Article

\title{
Exploiting the Chiral Ligands of Bis(imidazolinyl)- and Bis(oxazolinyl)thiophenes-Synthesis and Application in Cu-Catalyzed Friedel-Crafts Asymmetric Alkylation
}

\author{
Mohammad Shahidul Islam ${ }^{1, *(\mathbb{D})}$, Abdullah Saleh Alammari ${ }^{1} \mathbb{D}$, Assem Barakat ${ }^{1} \mathbb{D}$, Saeed Alshahrani ${ }^{1}$, \\ Matti Haukka ${ }^{2}$ (D) and Abdullah Mohammed Al-Majid ${ }^{1, *}$ \\ 1 Department of Chemistry, College of Science, King Saud University, P.O. Box 2455, \\ Riyadh 11451, Saudi Arabia; alamary1401@yahoo.com or 436106737@student.ksu.edu.sa (A.S.A.); \\ ambarakat@ksu.edu.sa (A.B.); chemistry99y@gmail.com or 436106738@student.ksu.edu.sa (S.A.) \\ 2 Department of Chemistry, University of Jyväskylä, P.O. Box 35, FI-40014 Jyväskylä, Finland; \\ matti.o.haukka@jyu.fi (M.H.) \\ * Correspondence: mislam@ksu.edu.sa (M.S.I.); amajid@ksu.edu.sa (A.M.A-M.)
}

check for updates

Citation: Islam, M.S.; Alammari, A.S.; Barakat, A.; Alshahrani, S.; Haukka, M.; Al-Majid, A.M. Exploiting the Chiral Ligands of Bis(imidazolinyl)- and

Bis(oxazolinyl)thiophenes-Synthesis and Application in Cu-Catalyzed Friedel-Crafts Asymmetric Alkylation. Molecules 2021, 26, 7408 https://doi.org/10.3390/ molecules26237408

Academic Editor: Alexander Viktorovich Aksenov

Received: 2 November 2021 Accepted: 3 December 2021 Published: 6 December 2021

Publisher's Note: MDPI stays neutral with regard to jurisdictional claims in published maps and institutional affiliations.

Copyright: (c) 2021 by the authors. Licensee MDPI, Basel, Switzerland. This article is an open access article distributed under the terms and conditions of the Creative Commons Attribution (CC BY) license (https:/ / creativecommons.org/licenses/by/ $4.0 /)$.

\begin{abstract}
Five new $C_{2}$-symmetric chiral ligands of 2,5-bis(imidazolinyl)thiophene (L1-L3) and 2,5bis(oxazolinyl)thiophene (L4 and L5) were synthesized from thiophene-2,5-dicarboxylic acid (1) with enantiopure amino alcohols $(\mathbf{4 a - c})$ in excellent optical purity and chemical yield. The utility of these new chiral ligands for Friedel-Crafts asymmetric alkylation was explored. Subsequently, the optimized tridentate ligand $\mathrm{L} 5$ and $\mathrm{Cu}(\mathrm{OTf})_{2}$ catalyst $(15 \mathrm{~mol} \%)$ in toluene for $48 \mathrm{~h}$ promoted Friedel-Crafts asymmetric alkylation in moderate to good yields (up to $76 \%$ ) and with good enantioselectivity (up to $81 \%$ ee). The bis(oxazolinyl)thiophene ligands were more potent than bis(imidazolinyl)thiophene analogues for the asymmetric induction of the Friedel-Crafts asymmetric alkylation.
\end{abstract}

Keywords: bis-oxazoline; bis-imidazoline; thiophene; indoles; $\beta$-nitroolefins; asymmetric catalysis; Friedel-Crafts alkylation

\section{Introduction}

Metal-catalyzed asymmetric transformation has become one of the most desirable strategies in advanced synthetic chemistry to access a variety of enantiopure organic molecules [1-8]. The optically active system can be achieved by means of various methodologies, such as chiral ligands assisted organocatalysis [9-11] and enzyme-catalyzed asymmetric conversion [12-14]. In addition, more advanced and refined approaches have been introduced effectively, such as stereo-convergent [15-17] and stereo-divergent synthesis [18-21] in order to acquire innumerable chiral frameworks.

Chiral ligand-Lewis acid metal complex-catalyzed asymmetric Friedel-Crafts alkylation reactions play a pivotal role in synthetic organic chemistry for the construction of new $\mathrm{C}-\mathrm{C}$ bonds [22-26]. During the past few years, several chiral bidendate ligands have been developed and used in the Lewis acid metal-catalyzed asymmetric FriedelCrafts alkylation reaction of indole with various substrates, including $\alpha, \beta$-unsaturated-Rketoesters $(\mathrm{R}=$ alkyl, aryl) $[27,28]$, $\mathrm{R}$-hydroxy enones $(\mathrm{R}=$ alkyl, aryl $)[29,30]$, alkylidene malonates [31-33], acyl phosphonates [34,35], acyl heterocyclic compounds [36-38], Nsulfonyl aldimines catalyzed by Schiff base complexes of $\mathrm{Cu}$ (II)-chiral amino alcohol [39], $\alpha$-trifluoromethylated $\beta$-nitrostyrenes catalyzed by chiral BINOL metal phosphate [40], nitroolefins catalyzed by oxazoline-imidazoline-Zn(II) [41], bis(oxazolinyl)-Cu(II) [42] and 2,5-bis(oxazolinyl)thiophenes- $\mathrm{Cu}$ (II) complexes [43]. Very recently, Tanaka et al. have documented homochiral metal-organic framework-catalyzed enantioselective Friedel-Crafts alkylation of $N, N$-dialkylanilines with trans- $\beta$-nitrostyrene [44]. That being said, very few 
examples of chiral metal-box-bis(oxazoline)/bis(imidazoline) complex-catalyzed enantioselective Friedel-Crafts alkylation of indole with nitroolefins have been documented to date [41,45-47].

In recent years, the application of nitroolefins as electrophiles has also been gaining notable interest among pharmacists due to the activation functionality of the nitro groups, which facilitate easy conversion to other useful functional groups to achieve numerous eyecatching chemical entities [48,49]. Furthermore, optically active Friedel-Crafts-alkylated product of indole with nitroolefins can also serve as an antecedent for the preparation of various drug molecules such as physostigmine [50,51], which acts as a clinically active anticholinergic drug [52], Recently, some examples of nitroalkenes have also been reported as Michael acceptors in metal-catalyzed asymmetric reaction due to the presence of strong electron-withdrawing nitro-groups $[48,53,54]$ e.g., rhodium-catalyzed additions of boronic acids to nitroalkenes [55], copper-catalyzed dialkylzinc additions to nitroalkenes [56,57], conjugated reductions of nitroalkenes [58] and the organo-catalyzed additions of 1,3dicarbonyl compounds to nitroalkenes [59,60].

Moreover, to date, most of the research work has been done with the main family of chiral ligands predominantly belonging to di-phosphine, diamine, di-ol, etc., i.e., phosphorous-, nitrogen- and oxygen-containing substrate. Very little research has been done in the recent past on developing chiral ligands based on sulfur-containing compounds. Therefore, researchers are highly interested in developing new chiral ligands based on a sulfur-containing moiety due to their high coordination ability to the most of the transition metals [61]. The sulfur atom is also considered as a soft atom that can bind strongly to soft metals, in particular copper metal $\mathrm{Cu}$ (II). In addition, sulfur-containing ligands are poor $\pi$-acceptors and poor $\sigma$-donors as compared to phosphine ligands, resulting in strong metal-sulfur bond strength. However, sulfur-containing ligand precursors are easily available, having extra advantages such as easy storage due to their higher tolerance to air as compared to phosphine-containing ligands, which makes them highly stable [61].

Recently, chiral ligand-Lewis acid-catalyzed asymmetric induction of indole with prochiral $\beta$-nitroolefin has become one of the most significant and successful pathways for accessing highly functionalized optically pure building blocks. Our research group has reported a new catalytic system based on the $\mathrm{Cu}(\mathrm{II})$ metal/chiral thiophene-2,5-bis $(\beta$ amino alcohol) ligands for an asymmetric Henry reaction of nitromethane with aromatic aldehyde with excellent ee (up to 94.6\%) and chemical yield (up to 99\%) [62]. In continuation of our research program, therefore, the design and synthesis of novel chiral 2,5-bis(imidazolinyl)thiophene and 2,5-bis(oxazolinyl)thiophene box-type ligands and their applications in various asymmetric catalyses remains a remarkable and interesting research topic to organic chemists. However, chiral ligands based on 2,5-bis(imidazolinyl)thiophene and 2,5-bis(oxazolinyl)thiophene framework could also be advantageous for several asymmetric transformations other than Friedel-Crafts alkylation reactions, such as asymmetric Henry reactions $[63,64]$, Diels-Alder reactions [65,66], enantioselective additions of diethylzinc to acyclic enones [67-69], asymmetric allylic substitutions [70,71] and asymmetric cyclopropanation $[72,73]$ reactions, etc. Keeping in mind the wide range of chiral applications of 2,5-bis(imidazolinyl)thiophene and 2,5-bis(oxazolinyl)thiophene box-type ligands and the diverse functionality of nitroolefins, we have decided to focus on this particular research field.

In this research article, we report the synthesis of novel chiral ligands thiophene2,5-2,5-bis(imidazolinyl)thiophene (L1-L3) and thiophene-2,5-bis(oxazolinyl)thiophene (L4 and L5) and their applications in Lewis acid metal-catalyzed asymmetric Friedel-Crafts alkylations of indole with electron-deficient prochiral $\beta$-nitroolefins.

Figure 1 shows some of the previously reported potent ligand structures used for asymmetric Friedel-Crafts alkylation reactions of indole with $\beta$-nitrostyrenes $[41,42,62,74-78]$. 


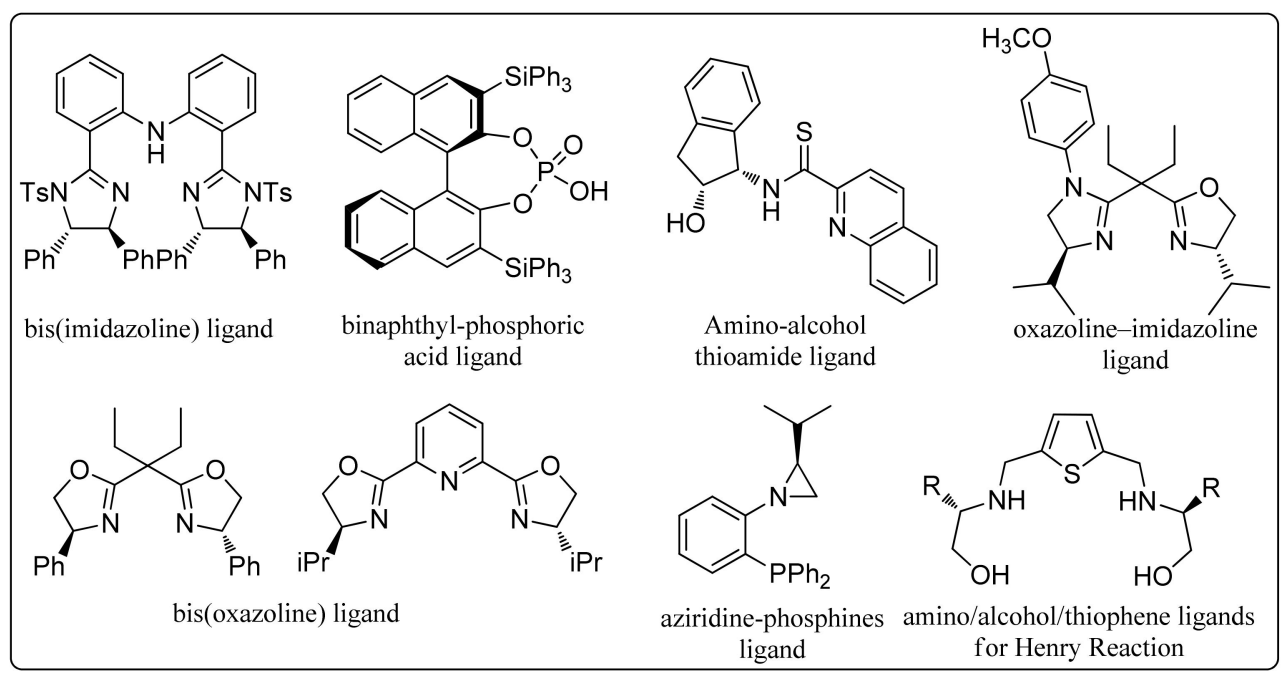

Figure 1. Previously reported potent ligand structures for asymmetric FC reaction.

\section{Results and Discussion}

2.1. Synthesis of chiral 2,5-bis(imidazolinyl)thiophene (L1-L3) and 2,5-bis(oxazolinyl)thiophene (L4 and L5)

Two set of $C_{2}$-symmetric 2,5-bis(imidazolinyl)thiophene (L1-L3) and 2,5-bis(oxazolinyl) thiophene (L4 and L5) ligands, based on thiophene framework, were synthesized from readily available and cheap thiophene-2,5-dicarboxlyic acid (1) and chiral amino alcohols $(\mathbf{3 a}-\mathbf{c})$ using well-known procedures reported in the literature [79] in five steps, as shown in Scheme 1. At the very outset, thiophene-2,5-dicarboxlyic acid (1) was treated with thionylchloride $\left(\mathrm{SOCl}_{2}\right)$ in the presence of a catalytic amount of $N, N$-dimethylformamide (DMF 2-3 drops) under reflux for $24 \mathrm{~h}$, leading to the formation of acid chloride (2) in quantitative yields (crude), which was then allowed to react with three different amino alcohols $(3 \mathbf{a}-\mathbf{c})$ in the presence of excess triethylamine (TEA) in dichloromethane $\left(\mathrm{CH}_{2} \mathrm{Cl}_{2}\right)$ to produce thiophene-2,5-dicarboxamide alcohol derivatives $(\mathbf{4 a}-\mathbf{c})$ with overall excellent isolated yield (75-97\%). Thiophene-2,5-dicarboxamide alcohol (4a) was then refluxed in thionylchloride $\left(\mathrm{SOCl}_{2}\right)$ for $24 \mathrm{~h}$ to afford crude thiophene-2,5-dicarboxamide dichloride (5a), which served as an intermediate for the synthesis of our target ligands L1-L3, while thiophene-2,5-dicarboxamide alcohol $(\mathbf{4 b}-\mathbf{c})$ was chosen as the precursor for the synthesis of ligands L4 and L5 (Figure 2).

Ligands (L1-L3) were synthesized using the intermediate thiophene-2,5-dicarboxamide dichloride $5 \mathbf{a}(2.92 \mathrm{mmol})$ by the reaction of three different aromatic amines $\mathbf{6 a}-\mathbf{c}(2.5 \mathrm{mmol})$ (aniline $\mathbf{6 a}, p$-chloroaniline $\mathbf{6 b}, p$-toludine $\mathbf{6 c}$ ) in the presence of excessive triethylamine (12 eq.) to form a corresponding thiophene-2,5-dicarboxamide intermediate (7a-c), which underwent a ring closure reaction upon treatment with $15 \%$ aqueous sodium hydroxide $(\mathrm{NaOH})$ solution to form crude thiophene-2,5-bis(imidazolinyl)thiophene ligands (L1L3). Then, the ligands were further purified by column chromatography by eluting with EtOAc/petroleum ether $/ \mathrm{Et}_{3} \mathrm{~N}(v: v: v=75: 24: 1)$ to afford pure ligands L1-L3 (Scheme 1). The isolated yields of the ligands were found to be in the range of $35-40 \%$.

Under inert condition, ligands (L4 and L5), were prepared from thiophene-2,5-dicarb oxamide alcohol ( $4 \mathbf{b}$ and $4 \mathbf{c}$ ) by ring closure reaction upon being treated with tosylchoride (1.25 eq.) and triethylamine (4.0 eq.) in the presence of a catalytic amount of DMAP (cat. 0.1eq.) in dichloromethane $\left(\mathrm{CH}_{2} \mathrm{Cl}_{2}\right)$ after $48 \mathrm{~h}$ of stirring at room temperature. The ligands were then purified by column chromatography, using $95 \% \mathrm{CH}_{2} \mathrm{Cl}_{2} / \mathrm{CH}_{3} \mathrm{OH}$ as an eluent to afford pure ligands L4 and L5 (Scheme 1) with 60\% and 55\% isolated yield, respectively. The formations of the compound thiophene-2,5-dicarboxamide alcohol (3a) and all the ligands (L1-L5) were confirmed and characterized by NMR and mass spectroscopy analysis. 


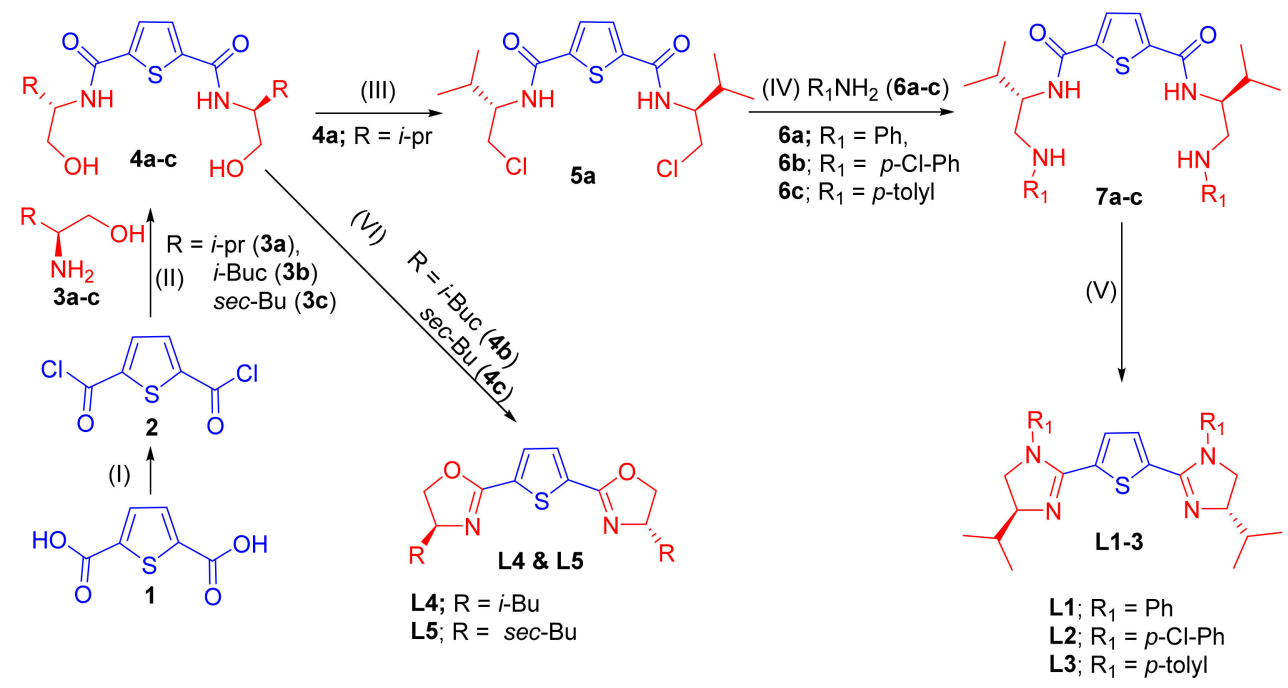

Scheme 1. 2,5-bis(imidazolinyl)thiophene (L1-L3) and 2,5-bis(oxazolinyl) thiophene (L4 and L5). Reaction conditions: (I) $\mathrm{SOCl}_{2}(8 \mathrm{~mL} / \mathrm{g})$, cat. DMF, $24 \mathrm{~h}$, reflux; (II) i. $\mathrm{CH}_{2} \mathrm{Cl}_{2}$, TEA (5 eq.), $-10{ }^{\circ} \mathrm{C}$; ii. Amino alcohol (3a-c) (2.1 eq.); (III) $4 \mathrm{a}$, $\mathrm{SOCl}_{2}(8.8 \mathrm{~mL} / \mathrm{g})$, refluxed, 24 h; (IV) i. $5 \mathbf{a}, \mathrm{Et}_{2} \mathrm{O}$, TEA (12.0 eq.), $0{ }^{\circ} \mathrm{C}$; ii. 2.5 eq. $\mathrm{R}_{1} \mathrm{NH}_{2}(\mathbf{6 a}-\mathrm{c}), 0{ }^{\circ} \mathrm{C}$, then r.t., $12 \mathrm{~h}$; $(\mathrm{V})$ $\mathrm{NaOH}$ (15\% aq. soln., $15 \mathrm{~mL} / \mathrm{g})$, r.t, 24 h; (VI) 4b-4c, Tosylchloride (1.25 eq.), DMAP (cat. 0.1 eq.), TEA (4.0 eq.), $\mathrm{CH}_{2} \mathrm{Cl}_{2}, \mathrm{r.t}$, $48 \mathrm{~h}, \mathrm{~N}_{2}$.
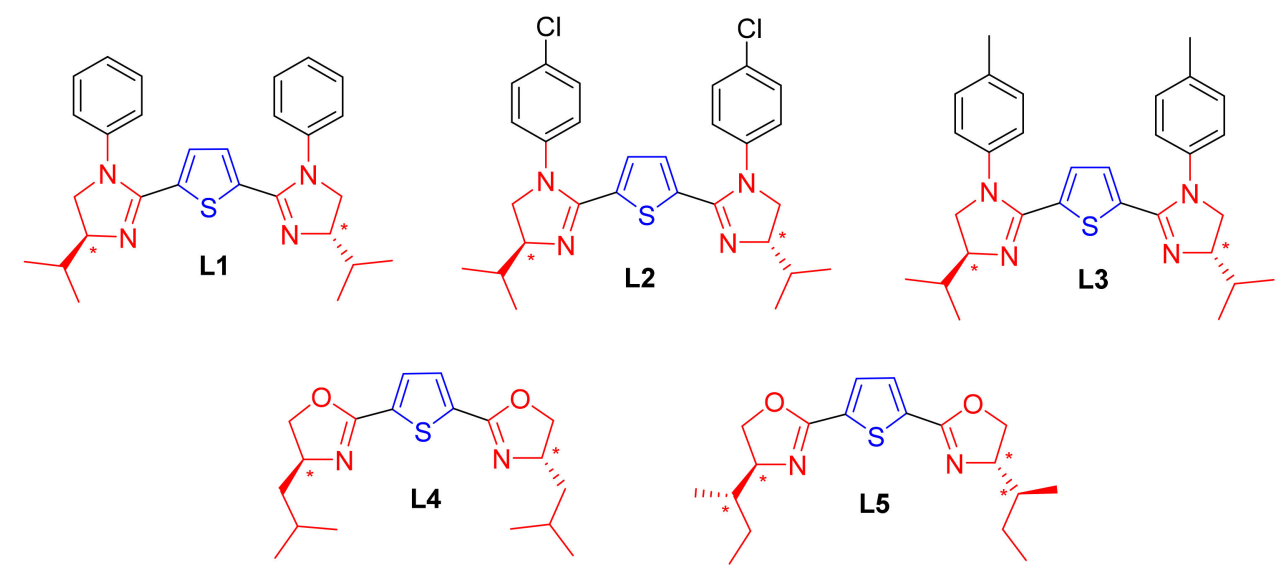

Figure 2. $C_{2}$-symmetric 2,5-bis(imidazolinyl)thiophene (L1-L3) and ligands 2,5-bis(oxazolinyl)thiophene (L4 and L5) tested for the Friedel-Crafts alkylation reaction of indoles with trans- $\beta$-nitrostyrene derivatives.

\subsection{Application of Chiral Ligand (L1-L5)}

2.2.1. Catalytic asymmetric Friedel-Crafts Alkylation of Indoles with Trans- $\beta$-nitrostyrene Derivatives; Optimization of Various Reaction Parameters

As soon as we had in our hand optically pure ligands L1-L5, we decided to carry out the catalytic activity in an asymmetric Friedel-Crafts alkylation reaction between indoles $\mathbf{8 a}-\mathbf{d}$ and nitrostyrene derivatives $\mathbf{9 a}-\mathbf{h}$. Indole (8a) and $p$-fluoronitrostyrene $(\mathbf{9 a})$ have been chosen as a model substrate for the reaction parameters optimization. In order to identify the best ligands for the asymmetric catalysis, initially, the Friedel-Crafts alkylation reaction of indole (8a) and $p$-fluoronitrostyrene $(\mathbf{9 a})$ was performed with the screened chiral bis(imidazoline) and bis(oxazoline) ligands L1-L5 (15 mol\%) and $\mathrm{Cu}(\mathrm{OTf})_{2}(15 \mathrm{~mol} \%)$ as metal sources in toluene at room temperature for $48 \mathrm{~h}$, and the subsequent findings are documented in Table 1. It is evident from the results summarized in Table 1, entries 1-5, that the thiophene-2,5-bis(oxazoline) ligand $\mathbf{L} 5$ performed very well under the above-mentioned reaction conditions and afforded Friedel-Crafts alkylation adduct 10a at $66 \%$ chemical yield with $75 \%$ enantiomeric excess (ee) (Table 1; entry 5), while ligand $\mathbf{L} 4$ yielded $70 \%$ chemical yield with $45 \%$ ee (Table 1; entry 4). Although the ligands L1-L3 furnished better 
chemical yields $(78,75$ and $70 \%$, respectively), only trace enantiomeric excess (ee) (3-5\%) was achieved (Table 1, entries 1-3). In order to improve the chemical yield, the reaction was repeated with ligand $\mathbf{L} 5$, and reaction time was extended up to $72 \mathrm{~h}$, but no significant changes were observed (Table 1; entry 6). Aiming to improve the chemical yield as well as enantioselectivity output of the reaction, a set of trials was conducted by variation of the loading of catalyst L5:Cu(OTf $)_{2}$ at 5, 10 and $20 \mathrm{~mol} \%$. The results showed that regardless of the $\%$ catalyst loading, the chemical yield was lower $(20 \%, 46 \%$ and $65 \%$, respectively) and did not result in any significant changes for the enantioselectivity $(65 \%, 71 \%$ and $74 \% e e)$ (Table 1, entries 7-9). The influences of the solvent effects were also studied; Friedel-Crafts alkylation reactions of indole (8a) and $p$-fluoronitrostyrene $(\mathbf{9 a})$ were also performed using a ligand-metal ratio of $15 \mathrm{~mol} \%$ of $\mathbf{L} 5: \mathrm{Cu}(\mathrm{OTf})_{2}$ at room temperature in several solvents, such as tetrahydrofuran, methanol, acetonitrile, dichloromethane, $n$-hexane and ethylacetate, within various time frames (84-96 h) (Table 1, entries 10-15), where dichloromethane was found to be the best solvent for chemical yield improvement but with no enantioselectivity (Table 1; entry 13), whereas no product formation took place in $n$-hexane and ethylacetate (Table 1, entries 14 and 15), although in THF, moderate yield (48\%) and enantioselectivity $(55 \%)$ were observed (Table 1 ; entry 10$)$. From the above preliminary findings, it is obvious that a $15 \mathrm{~mol} \%$ ligand-metal ratio [ $\left.15 \mathrm{~mol} \% \mathrm{~L} 5: \mathrm{Cu}(\mathrm{OTf})_{2}\right]$ in toluene at room temperature in $48 \mathrm{~h}$ was the optimum set of reaction conditions to afford the final $\mathrm{C}-\mathrm{C}$ bond formation adduct. Interestingly, it is clear from the preliminary results that oxazolinyl-based ligands are more potent than imidazolinyl-based ones; more interestingly, the substitution at the oxazolinyl moiety showed to also be critical for the asymmetric induction. Further investigation for better understanding is highly recommended.

Table 1. Friedel-Crafts alkylation reaction of indole (8a) with $p$-fluoronitrostyrene (9a) as model substrate; reaction optimization (ligands, solvents and time).

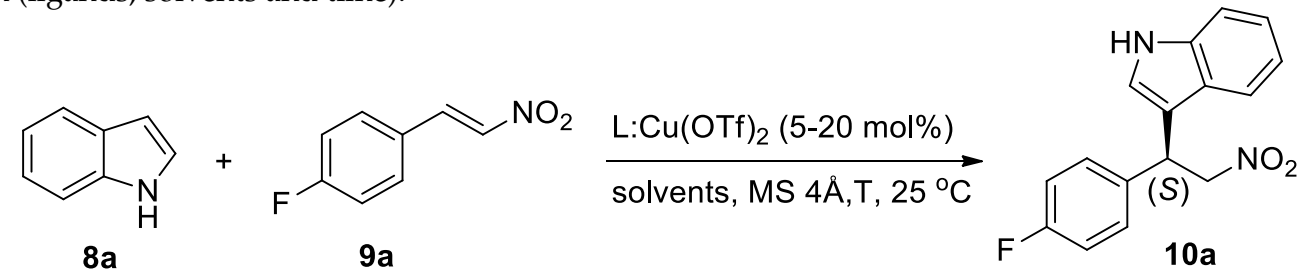

\begin{tabular}{|c|c|c|c|c|c|c|}
\hline Entry $^{[a]}$ & Ligands & $\mathrm{L}: \mathrm{Cu}(\mathrm{OTf})_{2}[1: 1]$ & Solvents & Time [h] & Yield $(\%)^{[b]}$ & $e e(\%)^{[c, d]}$ \\
\hline 1. & L1 & $15 \mathrm{~mol} \%$ & Toluene & 48 & 78 & 5 \\
\hline 2. & L2 & $15 \mathrm{~mol} \%$ & Toluene & 48 & 75 & 3 \\
\hline 3. & L3 & $15 \mathrm{~mol} \%$ & Toluene & 48 & 77 & 3 \\
\hline 4. & L4 & $15 \mathrm{~mol} \%$ & Toluene & 48 & 70 & 45 \\
\hline 5. & L5/, & $15 \mathrm{~mol} \%$ & Toluene & 48 & 66 & 75 \\
\hline 6. & L5 & $15 \mathrm{~mol} \%$ & Toluene & 72 & 68 & 74 \\
\hline 7. & L5 & $5 \mathrm{~mol} \%$ & Toluene & 48 & 20 & 65 \\
\hline 8. & L5 & $10 \mathrm{~mol} \%$ & Toluene & 48 & 46 & 71 \\
\hline 9. & L5 & $20 \mathrm{~mol} \%$ & Toluene & 48 & 65 & 74 \\
\hline 10. & L5 & $15 \mathrm{~mol} \%$ & THF & 48 & 55 & 50 \\
\hline 11. & L5 & $15 \mathrm{~mol} \%$ & $\mathrm{MeOH}$ & 72 & 30 & 5 \\
\hline 12. & L5 & $15 \mathrm{~mol} \%$ & $\mathrm{ACN}$ & 96 & 10 & 4 \\
\hline 13. & L5 & $15 \mathrm{~mol} \%$ & DCM & 72 & 80 & 0 \\
\hline 14. & L5 & $15 \mathrm{~mol} \%$ & Hexane & 72 & - & - \\
\hline 15. & L5 & $15 \mathrm{~mol} \%$ & EA & 96 & traces & - \\
\hline
\end{tabular}

${ }^{[a]}$ All the reactions were conducted on a $0.2 \mathrm{mmol}$ scale; ${ }^{[b]}$ isolated yields after column purification; ${ }^{[c]}$ the enantiomeric excess (ee) was measured by chiral HPLC using a Daicel OD-H column $(25 \mathrm{~cm} \times 4.6 \mathrm{~mm} \times 5 \mu \mathrm{m})$; ${ }^{[\mathrm{d}]}$ the absolute configuration was assigned as $(S)$ comparing the retention time and sign of optical rotation reported in the literature [74].

Next, another two factors were also investigated, namely metal salts and temperature effects. Therefore, a Friedel-Crafts alkylation of indole (8a) with $p$-fluoronitrostyrene (9a) was carried out using $15 \mathrm{~mol} \%$ of ligand $\mathrm{L} 5$ with the combination of several metal triflates, such as $\mathrm{Zn}(\mathrm{OTf})_{2}, \mathrm{Mg}(\mathrm{OTf})_{2}, \operatorname{Er}(\mathrm{OTf})_{2}$ and $\mathrm{Yb}(\mathrm{OTf})_{2}$, and metal chlorides such 
as $\mathrm{FeCl}_{3}$ and $\mathrm{PdCl}_{2}$, in toluene at $25{ }^{\circ} \mathrm{C}$, and the results are summarized in Table 2. It was observed from the metal screening that $\mathrm{Zn}(\mathrm{OTf})_{2}, \mathrm{FeCl}_{3}$ and $\mathrm{PdCl}_{2}$ yielded product 10a with excellent to good chemical yields $(97 \%, 80 \%$ and $70 \%$, respectively), while the enantioselectivity remains negligible (Table 2, entries 1, 5 and 6). Two attempts were carried out at low $\left(0{ }^{\circ} \mathrm{C}\right)$ and high $\left(70{ }^{\circ} \mathrm{C}\right)$ temperature for $92 \mathrm{~h}$ and $24 \mathrm{~h}$, respectively, and henceforth, $42 \%$ and $70 \%$ chemical yields with $76 \%$ and $65 \%$ enantioselectivity were observed (Table 2, entries 7 and 8). The results showed no significant changes for either the chemical yield or the enantioselectivity (Table 2, entry 8). From the overall findings, a catalyst generated in situ from ligand $\mathbf{L} 5$ and Lewis acid $\mathrm{Cu}(\mathrm{OTf})_{2}$ in toluene was found to be the optimum reaction condition for the asymmetric Friedel-Crafts alkylation of indole (8a) and $p$-fluoronitrostyrene (9a).

Table 2. Friedel-Crafts arylation of indole (8a) with $p$-fluoronitrostyrene (9a) as model substrate reaction optimization (temperature and metals salts).

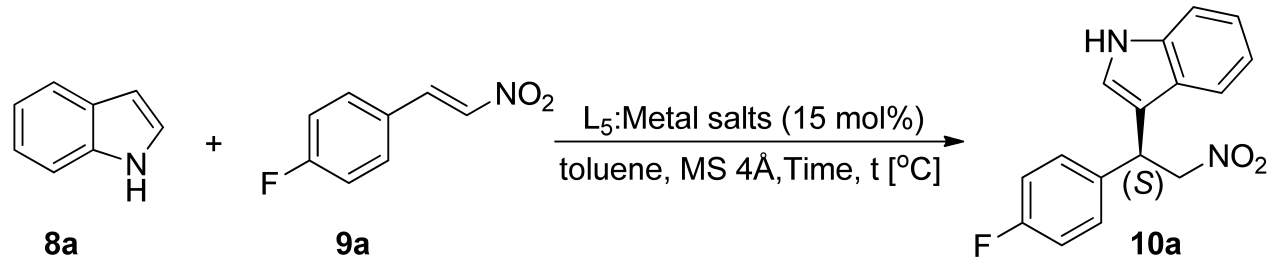

\begin{tabular}{|c|c|c|c|c|c|}
\hline Entry $^{[a]}$ & Metals Salts (15 mol\%) & Time [h] & Temp $\left[{ }^{\circ} \mathrm{C}\right]$ & Yield (\%) ${ }^{[b]}$ & $e e(\%){ }^{[c, d]}$ \\
\hline 1. & $\mathrm{Zn}(\mathrm{OTf})_{2}$ & 48 & 25 & 97 & 10 \\
\hline 2. & $\operatorname{Mg}(\mathrm{OTf})_{2}$ & 72 & 25 & - & - \\
\hline 3. & $\operatorname{Er}(\mathrm{OTf})_{2}$ & 72 & 25 & 40 & 2 \\
\hline 4. & $\mathrm{Yb}(\mathrm{OTf})_{2}$ & 72 & 25 & 47 & 0 \\
\hline 5. & $\mathrm{FeCl}_{3}$ & 24 & 25 & 80 & 2 \\
\hline 6. & $\mathrm{PdCl}_{2}$ & 24 & 25 & 70 & 0 \\
\hline 7. & $\mathrm{Cu}(\mathrm{OTf})_{2}$ & 92 & 0 & 42 & 76 \\
\hline 8. & $\mathrm{Cu}(\mathrm{OTf})_{2}$ & 24 & 70 & 66 & 65 \\
\hline
\end{tabular}

${ }^{[\mathrm{a}]}$ All the reactions were conducted on a $0.2 \mathrm{mmol} \mathrm{scale;}{ }^{[\mathrm{b}]}$ isolated yields after column purification; ${ }^{[c]}$ the enantiomeric excess (ee) was measured by chiral HPLC using a Daicel OD-H column $(25 \mathrm{~cm} \times 4.6 \mathrm{~mm} \times 5 \mu \mathrm{m})$; ${ }^{[\mathrm{d}]}$ the absolute configuration was assigned as $(S)$ comparing the retention time and sign of optical rotation reported in the literature [74].

\subsubsection{Substrate Scope}

To illustrate the generality, 20 examples of asymmetric Friedel-Crafts alkylation reactions have been carried out using indoles $\mathbf{8 a}-\mathbf{d}$ with various nitroolefins $(\mathbf{9 a}-\mathbf{h})$ under the optimized reaction conditions, i.e., $15 \mathrm{~mol} \% \mathrm{~L} 5: \mathrm{Cu}(\mathrm{OTf})_{2}$ in toluene at $25{ }^{\circ} \mathrm{C}$ for $48 \mathrm{~h}$, and the results are shown in Table 3. After the observing the results, it seems that substrates 9a-h reacted with indole 8a moderately and yielded chiral products $10 \mathbf{a}-\mathbf{f}$ in the range of $40-67 \%$ yields with $64-80 \%$ enantioselectivity. Substrates $9 \mathbf{a}, \mathbf{9 b}, \mathbf{9 d}, \mathbf{9 e}$ and $\mathbf{9 h}$ performed fairly well, yielding corresponding FC products 10a, 10b, 10d, 10e and 10h with 67, 64, 66, 58 and $60 \%$ yields and good enantiomeric excess (ee) at 74, 80, 69, 70 and $64 \% e e$, respectively (Table 3, entries 1, 2, 4, 5 and 8). While substrates $\mathbf{9 c}$, 9 f and $\mathbf{9 g}$ furnished the corresponding Friedel-Crafts alkylated products 10c, 10f and $10 \mathrm{~g}$ with poor chemical yields $(40,48$ and $52 \%$, respectively) because of the steric hindrance of the substrate, the enantioselectivity remained good (75, 71 and 71\%, respectively) (Table 3, entries 3, 6 and 7). When substrate $\mathbf{9 a}-\mathbf{h}$ was allowed to react with 5-bromoindole (8b) under the optimized conditions, poor yields were observed (10i-p, 35-55\%) with good enantioselectivity (60-81\% ee) (Table 3, entries 9-16). A Friedel-Crafts reaction of 5-fluoro indole with $\beta$-nitrostyrene $9 \mathrm{~g}$ furnished a moderate yield $(57 \%)$ with good enantioselectivity $(66 \% е e)$ as compared to the reaction with the more hindered $9 \mathbf{h}$, which produced poor yield $(45 \%)$ as well as poor enantioselectivity $(21 \%$ ee) (Table 3, entries 17 and 18). We further performed the Friedel-Crafts reaction with $N$-ethyl-protected indole and $\beta$-nitrostyrene $9 \mathbf{a}$ and $\mathbf{9 d}$, which produced good yields (73 and 76\%) with poor enantiomeric excess (35 and 27\%) (Table 3, entries 19 and 20). 
Interestingly, when the asymmetric Friedel-Crafts alkylation of indole 8a with nitrostyrene 9a was performed at a large scale (10-fold), both the yield (76\%) and enantioselectivity (77\% ee) were improved (Table 3, entry 1).

Table 3. Substrate scope by reaction of indole derivatives (8a-d) with substituted nitrostyrene (9a-h) under optimized reaction condition.
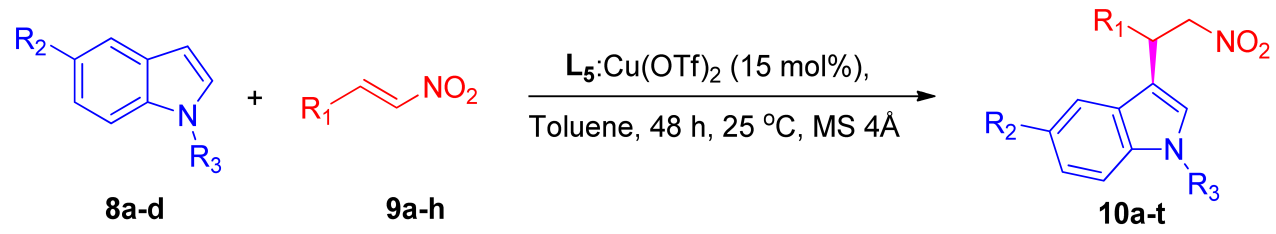

\begin{tabular}{|c|c|c|c|c|c|c|c|c|}
\hline Entry $^{[a]}$ & $R_{1}(9 a-h)$ & $\mathbf{R}_{2}$ & $\mathbf{R}_{3}$ & $10 a-i$ & Yields (\%) ${ }^{[b]}$ & $e e(\%){ }^{[c]}$ & $R / S$ & Ref. \\
\hline 1. & $4-\mathrm{F}-\mathrm{C}_{6} \mathrm{H}_{4}$ & $\mathrm{H}$ & $\mathrm{H}$ & $10 a$ & $6776^{[\mathrm{LS}]}$ & $7477^{[\mathrm{LS}]}$ & $(S)^{[\mathrm{d}]}$ & [74] \\
\hline 2. & $3-\mathrm{Br}-\mathrm{C}_{6} \mathrm{H}_{4}$ & $\mathrm{H}$ & $\mathrm{H}$ & $10 \mathrm{~b}$ & 64 & 80 & $(S)[\mathrm{d}]$ & {$[74,76]$} \\
\hline 3. & $4-\mathrm{CF}_{3}-\mathrm{C}_{6} \mathrm{H}_{4}$ & $\mathrm{H}$ & $\mathrm{H}$ & $10 c$ & 40 & 75 & $(S)^{[\mathrm{d}]}$ & [75] \\
\hline 4. & $4-\mathrm{CH}_{3} \mathrm{O}-\mathrm{C}_{6} \mathrm{H}_{4}$ & $\mathrm{H}$ & $\mathrm{H}$ & $10 d$ & 66 & 69 & $(S)[\mathrm{d}]$ & [74] \\
\hline 5. & $2-\mathrm{NO}_{2}-\mathrm{C}_{6} \mathrm{H}_{4}$ & $\mathrm{H}$ & $\mathrm{H}$ & $10 \mathrm{e}$ & 58 & 70 & $(R)^{[\mathrm{d}]}$ & [80] \\
\hline 6. & $2,4-\mathrm{Cl}_{2}-\mathrm{C}_{6} \mathrm{H}_{3}$ & $\mathrm{H}$ & $\mathrm{H}$ & $10 f$ & 48 & 71 & $(R)^{[\mathrm{d}]}$ & [74] \\
\hline 7. & 2-thienyl & $\mathrm{H}$ & $\mathrm{H}$ & $10 \mathrm{~g}$ & 52 & 71 & $(S)[\mathrm{e}]$ & [42] \\
\hline 8. & $2,6-\mathrm{Cl}_{2}-\mathrm{C}_{6} \mathrm{H}_{3}$ & $\mathrm{H}$ & $\mathrm{H}$ & $10 \mathrm{~h}$ & 60 & 64 & $(R)^{[\mathrm{e}]}$ & [41] \\
\hline 9. & $4-\mathrm{F}-\mathrm{C}_{6} \mathrm{H}_{4}$ & $\mathrm{Br}$ & $\mathrm{H}$ & $10 \mathrm{i}$ & 55 & 77 & $(S)[\mathrm{e}]$ & \\
\hline 10. & $3-\mathrm{Br}-\mathrm{C}_{6} \mathrm{H}_{4}$ & $\mathrm{Br}$ & $\mathrm{H}$ & $10 \mathrm{j}$ & 46 & 81 & $(S)[\mathrm{e}]$ & \\
\hline 11. & $4-\mathrm{CF}_{3}-\mathrm{C}_{6} \mathrm{H}_{4}$ & $\mathrm{Br}$ & $\mathrm{H}$ & $10 \mathrm{k}$ & 35 & 79 & $(S)[\mathrm{e}]$ & \\
\hline 12. & $4-\mathrm{CH}_{3} \mathrm{O}-\mathrm{C}_{6} \mathrm{H}_{4}$ & $\mathrm{Br}$ & $\mathrm{H}$ & 101 & 39 & 63 & $(S)^{[\mathrm{d}]}$ & [81] \\
\hline 13. & $2-\mathrm{NO}_{2}-\mathrm{C}_{6} \mathrm{H}_{4}$ & $\mathrm{Br}$ & $\mathrm{H}$ & $10 \mathrm{~m}$ & 42 & 78 & $(R)^{[\mathrm{e}]}$ & \\
\hline 14. & $2,4-\mathrm{Cl}_{2}-\mathrm{C}_{6} \mathrm{H}_{3}$ & $\mathrm{Br}$ & $\mathrm{H}$ & $10 n$ & 37 & 75 & $(R)^{[\mathrm{e}]}$ & \\
\hline 15. & 2-thienyl & $\mathrm{Br}$ & $\mathrm{H}$ & $10 o$ & 47 & 72 & $(S)[\mathrm{e}]$ & [42] \\
\hline 16. & $2,6-\mathrm{Cl}_{2}-\mathrm{C}_{6} \mathrm{H}_{3}$ & $\mathrm{Br}$ & $\mathrm{H}$ & $10 p$ & 52 & 60 & $(R)^{[\mathrm{e}]}$ & \\
\hline 17. & 2-thienyl & $\mathrm{F}$ & $\mathrm{H}$ & $10 q$ & 57 & 66 & $(S)[\mathrm{e}]$ & \\
\hline 18. & $2,6-\mathrm{Cl}_{2}-\mathrm{C}_{6} \mathrm{H}_{3}$ & $\mathrm{~F}$ & $\mathrm{H}$ & 10r & 45 & 21 & $(R)^{[\mathrm{e}]}$ & \\
\hline 19. & $4-\mathrm{F}-\mathrm{C}_{6} \mathrm{H}_{4}$ & $\mathrm{H}$ & Et & $10 \mathrm{~s}$ & 73 & 35 & $(S)^{[\mathrm{e}]}$ & \\
\hline 20. & $4-\mathrm{CH}_{3} \mathrm{O}-\mathrm{C}_{6} \mathrm{H}_{4}$ & $\mathrm{H}$ & Et & $10 t$ & 76 & 27 & $(S)^{[\mathrm{e}]}$ & [82] \\
\hline
\end{tabular}

${ }^{[a]}$ All the reactions were conducted on a $0.2 \mathrm{mmol} \mathrm{scale;}{ }^{[\mathrm{b}]}$ isolated yields after column purification; ${ }^{[c]}$ the $e e$ values were determined by chiral HPLC using a Daicel OD-H column $(25 \mathrm{~cm} \times 4.6 \mathrm{~mm} \times 5 \mu \mathrm{m})$ [74]; ${ }^{[\mathrm{d}]}$ the absolute configuration was determined as $(S)$ or $(R)$ comparing their retention time and sign of optical rotation reported in the literature; ${ }^{[\mathrm{e}]}$ the absolute configuration was assigned as $(S)$ or $(R)$ assuming uniform reaction mechanism and comparing with retention time and sign of optical rotation; ${ }^{[L S]}$ large-scale reaction yield and enantiomeric excess (ee).

Finally, to examine another nitrostyrene system for the Friedel-Crafts arylation, two nitrostyrene (9i and $\mathbf{9 j}$ )-based indole scaffold were synthesized and characterized. The synthesized indole-based nitrostyrenes $\mathbf{9} \mathbf{i}$ and $\mathbf{9} \mathbf{j}$ were used as substrates for the asymmetric Friedel-Crafts arylation using our optimized method, but they unfortunately did not succeed in affording the final desired chiral FC products 10u and 10v, as shown in Scheme 2. The requisite final compounds either did not occur or decomposed.

In Figure 3, the proposed cycle of the catalytic mechanism has been shown, where in the intermediates (II) and (III), it has been clearly shown that the addition of an incoming nucleophilic group from the $S i$ face is more favorable than the $R e$ face since the latter is a more sterically hindered face as compared to former. 


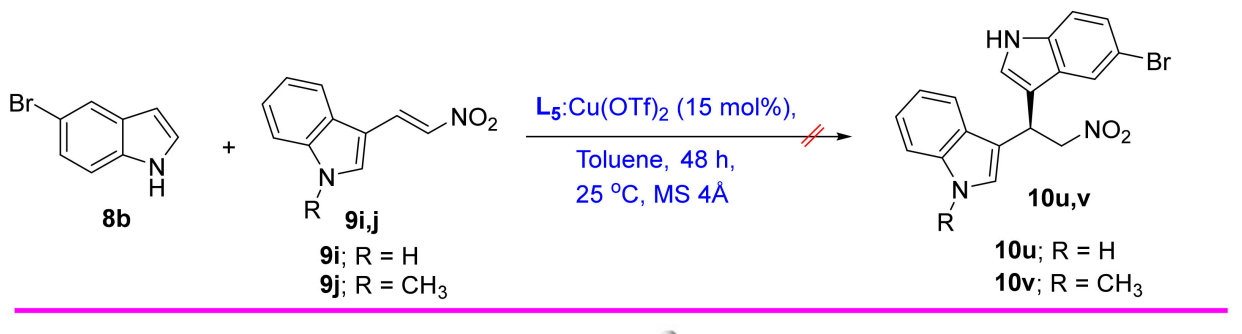

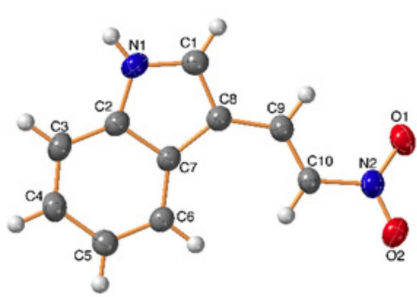

$9 \mathrm{i}$

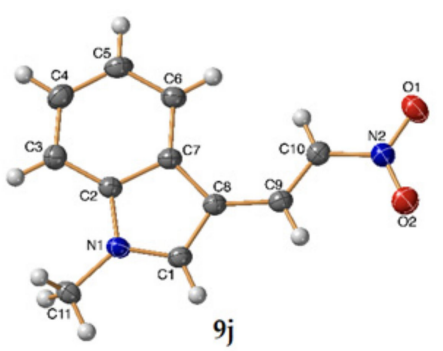

Scheme 2. Friedel-Crafts arylation of indole (8b) with nitrostyrene-based indole scaffold (9i and $\mathbf{9 j})$.

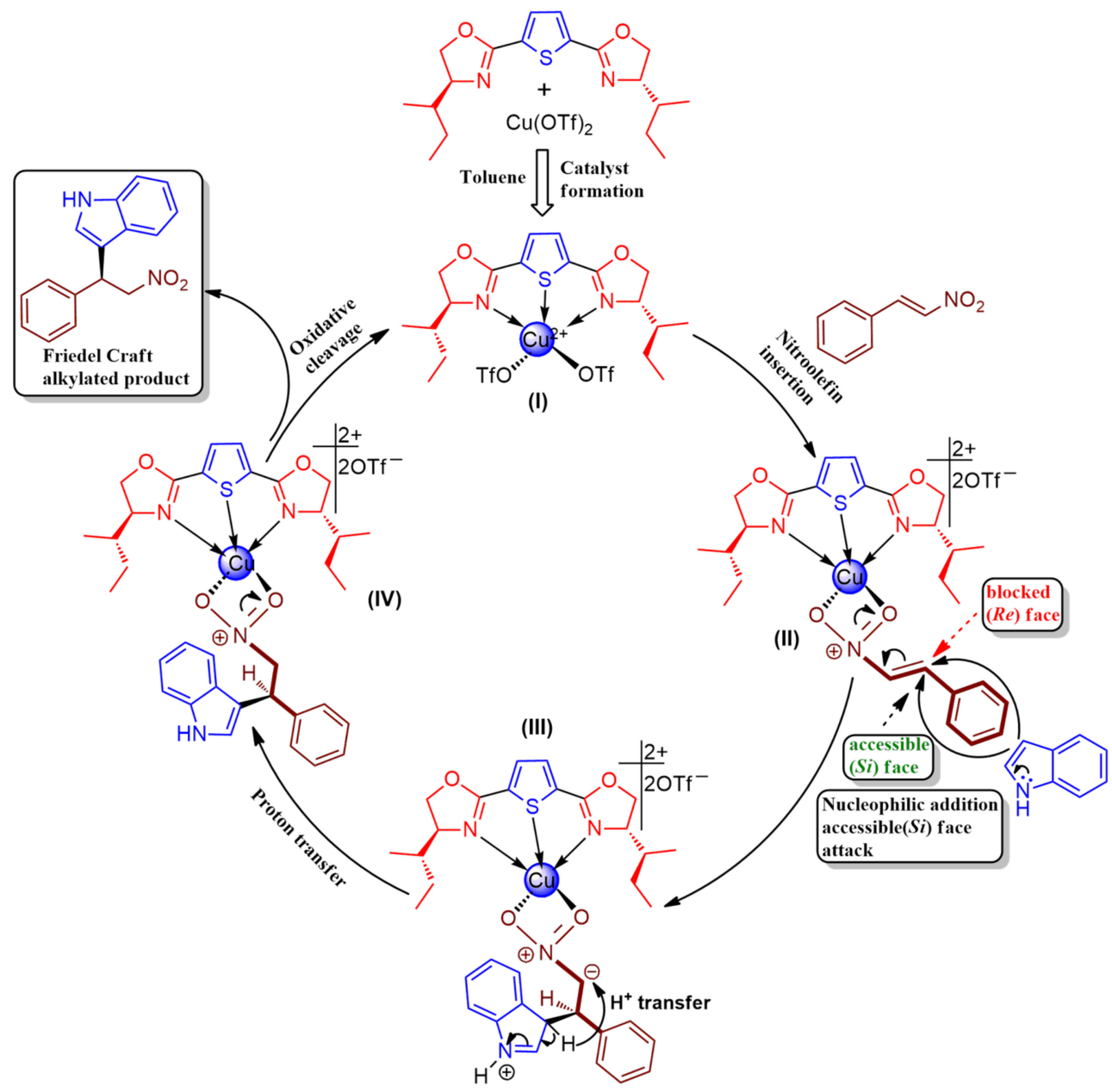

Figure 3. Proposed mechanism: L5:Cu(OTf $)_{2}$-catalyzed Friedel-Craft alkylation of indole with $\beta$-nitroolefin catalytic cycle.

In case of Friedel-Craft product with indole, the retention time of the $S$ enantiomer was found to be lesser than the $R$ enantiomer in the chiral HPLC analysis using Daicel OD-H chiral column and $n$-hexane/iso-propanol system in the reported literature, while for FC products with 5-bromoindole it was found to be vice versa. Therefore, the absolute configuration of the synthesized chiral FC products $10 \mathrm{a}-\mathrm{d}, 10 \mathrm{~g}, 10 \mathrm{i}-1,10 \mathrm{o}, 10 \mathrm{q}, 10 \mathrm{~s}$ and 
$10 \mathrm{t}$ was assigned as $S$, while 10e, 10f, 10h, 10m, 10n, 10p and 10r were assigned as $R$ by comparing their retention time and optical rotation values found in reported literature, assuming that the reaction took place via uniform mechanistic pathway (Table 3) [41,74].

\section{Materials and Methods}

\subsection{General}

Reagents obtained from commercial suppliers were used without further purification. Preparation of bis(imidazoline) and bis(oxazoline) ligands was performed in dried glassware flasks under a static pressure of nitrogen. Solvents were dried prior to use following standard procedures. Reactions were monitored by thin layer chromatography using Merck silica gel 60 Kieselgel F254 TLC (Merck, Kenilworth, NJ, USA), and column chromatography was performed on silica gel 100-200 (40-63 $\mu \mathrm{m}$, ASTM) from Merck using the indicated solvents. ${ }^{1} \mathrm{H}$ and ${ }^{13} \mathrm{C}-\mathrm{NMR}$ spectra were recorded in $\mathrm{CDCl}_{3}$ and DMSO- $d_{6}$ on a Jeol Spectrometer (Jeol, Tokyo, Japan) (400 MHz and $500 \mathrm{MHz}$ ). The chemical shifts are reported in ppm. All the racemic products were freshly prepared as per the method reported in the literature [83]. Infrared spectra were recorded on a Thermo Scientific Nicolet iS10 FT-IR spectrometer (Thermo Fisher Scientific, Waltham, MA, USA). Enantiomeric ratios were determined by analytical chiral HPLC analysis on a Shimadzu LC-20A (Shimadzu, Kyoto, Japan) Prominence instrument with a chiral stationary phase using Daicel OD-H columns (Chiral Technologies Europe, Illkirch-Graffenstaden, France) and 70-75\% n-hexane/iso-propanol as eluents (Supplementary Materials). Optical rotations were obtained with a PerkinElmer 343 Polarimeter (PerkinElmer, Waltham, MA, USA). Melting points (m.p.) were recorded on a Thomas-Hoover capillary melting point apparatus (Thomas-Hoover, Texas City, USA) and were not corrected. Mass spectrometric analysis was done using ESI mode on an Agilent Technologies 6410-triple quad LC/MS instrument (Agilent, Santa Clara, CA, USA). Elemental analyses were performed on Perkin-Elmer PE 2400 CHN Elemental Analyzer with autosampler, CHN mode. X-ray diffraction data were collected on a Rigaku Oxford Diffraction Supernova diffractometer and processed with CrysAlisPro software v. 1.171.41.93a (Rigaku Oxford Diffraction, Yarnton, UK, 2020) using $\mathrm{Cu}$ K_ radiation".

\subsection{General Procedure (GP1) for the Preparation of Bis(hydroxyamides) $4 \boldsymbol{a}-\boldsymbol{c}$}

GP1: A 100-mL round bottom flask was charged with thiophene-2,5-dicarboxlyic acid (1) $(0.5 \mathrm{mg}, 2.9 \mathrm{mmol})$ and $\mathrm{SOCl}_{2}(7 \mathrm{~mL})$. A catalytic amount of DMF (3 drops) was added, and the reaction was reflux for $24 \mathrm{~h}$ under inert atmosphere. The reaction was then cooled, and excess $\mathrm{SOCl}_{2}$ was removed under reduced pressure to give the corresponding crude acid chloride (2). The crude acid chloride $2(2.9 \mathrm{mmol})$ solution in $\mathrm{CH}_{2} \mathrm{Cl}_{2}(10 \mathrm{~mL})$ was then slowly added to a pre-stirred solution of amino alcohol 3a-c (6.9 mmol, 2.1 eq.) and triethylamine ( $2 \mathrm{~mL}, 5$ eq.) in $\mathrm{CH}_{2} \mathrm{Cl}_{2}(35 \mathrm{~mL})$ at $-10{ }^{\circ} \mathrm{C}$. The reaction was then stirred at ambient temperature for $24 \mathrm{~h}$. After reaction completion, the solvents were removed and the residue was poured into water $(55 \mathrm{~mL})$. Upon standing at room temperature for $4 \mathrm{~h}$, solid product was precipitated out, which was then collected by filtration and purified by column chromatography using 100-200 mesh silica gel and $\mathrm{CH}_{2} \mathrm{Cl}_{2} / \mathrm{MeOH}$ (95:5) as an eluent to afford pure products $4 \mathbf{a}-\mathbf{c}$.

\subsection{1. $N^{2}, N^{5}$-Bis((S)-1-Hydroxy-3-methylbutan-2-yl)thiophene-2,5-dicarboxamide (4a)}

Following GP1, thiophene-2,5-dicarboxlyic acid chloride (2) and (S)-2-amino-3-methyl butan-1-ol (3a) reacted to produce 2,5-dicarboxamide alcohol (4a) as white solid (0.74 g, 75\%); m.p. $199-201^{\circ} \mathrm{C} ;[\alpha]_{\mathrm{D}}^{20}=-26^{\circ}\left(c 0.20, \mathrm{CH}_{3} \mathrm{OH}\right) ; \mathrm{IR}\left(\mathrm{KBr}, \mathrm{cm}^{-1}\right): 3350,3086,3071,2956$, 2870, 2496, 1627, 1543, 1515, 1464, 1033, 743; ${ }^{1} \mathrm{H}-\mathrm{NMR}\left(400 \mathrm{MHz}, \mathrm{DMSO}-d_{6}\right): \delta(\mathrm{ppm})=8.11$ $(\mathrm{d}, J=8.9 \mathrm{~Hz}, 2 \mathrm{H}, \mathrm{NH}), 7.82(\mathrm{~s}, 2 \mathrm{H}, \mathrm{Ar}-\mathrm{H}), 4.63(\mathrm{t}, J=5.8 \mathrm{~Hz}, 2 \mathrm{H}, \mathrm{NHCH}), 3.74(\mathrm{p}, J=7.0$, $\left.6.4 \mathrm{~Hz}, \mathrm{CH}_{2} \mathrm{OH}\right), 3.56-3.45\left(\mathrm{~m}, 4 \mathrm{H}, \mathrm{CH}_{2} \mathrm{OH}\right), 1.91\left(\mathrm{dp}, J=13.3,6.2 \mathrm{~Hz}, 2 \mathrm{H}, \mathrm{CH}\left(\mathrm{CH}_{3}\right)_{2}\right)$, $0.88\left(\mathrm{dd}, J=11.5,6.7 \mathrm{~Hz}, 12 \mathrm{H}, \mathrm{CH}\left(\mathrm{CH}_{3}\right)_{2}\right) ;{ }^{13} \mathrm{C}-\mathrm{NMR}(101 \mathrm{MHz}, \mathrm{DMSO}-d 6) \delta(\mathrm{ppm})=160.8$, $143.5,128.1,61.2,56.9,28.6,19.6,18.7 ; \mathrm{LC} / \mathrm{MS}$ (ESI): found $342.2[\mathrm{M}+\mathrm{H}]^{+}, \mathrm{C}_{16} \mathrm{H}_{26} \mathrm{~N}_{2} \mathrm{O}_{4} \mathrm{~S}$ 
requires 342.16; anal. calcd. for $\mathrm{C}_{16} \mathrm{H}_{26} \mathrm{~N}_{2} \mathrm{O}_{4} \mathrm{~S}$ : C, 56.12; $\mathrm{H}, 7.65 ; \mathrm{N}, 8.18$; found: $\mathrm{C}, 55.88 ; \mathrm{H}$, $7.72 ; \mathrm{N}, 8.06$.

\subsection{2. $N^{2}, N^{5}$-Bis((S)-1-Hydroxy-4-methylpentan-2-yl)thiophene-2,5-dicarboxamide (4b)}

Following GP1, thiophene-2,5-dicarboxlyic acid chloride (2) and (S)-2-amino-4-methyl pentan-1-ol (3b) reacted to produce 2,5-dicarboxamide alcohol $(4 \mathbf{b})$ as white solid (1.02 g, 95\%); m.p. $208-210{ }^{\circ} \mathrm{C}$; $[\alpha]_{\mathrm{D}}^{20}=-40.36^{\circ}$ (c 0.11, $\left.\mathrm{CH}_{3} \mathrm{OH}\right) ; \mathrm{IR}\left(\mathrm{KBr}, \mathrm{cm}^{-1}\right)$ : 3351, 3087, 2958, 2871, 2605, 2498, 1627, 1545, 1517, 1469, 1033, 745; ${ }^{1} \mathrm{H}-\mathrm{NMR}$ (500 MHz, DMSO- $\left.d_{6}\right)$ : $\delta(\mathrm{ppm})=8.19(\mathrm{~d}, J=8.7 \mathrm{~Hz}, 2 \mathrm{H}, \mathrm{NH}), 7.77(\mathrm{~s}, 2 \mathrm{H}, \mathrm{Ar}-\mathrm{H}), 4.74(\mathrm{~s}, 2 \mathrm{H}, \mathrm{NHCH}), 4.04-3.91$ $\left(\mathrm{m}, 2 \mathrm{H}, \mathrm{CH}_{2} \mathrm{OH}\right), 3.41\left(\mathrm{dt}, J=11.0,5.7 \mathrm{~Hz}, 2 \mathrm{H}, \mathrm{CH}_{2} \mathrm{OH}\right), 3.05\left(\mathrm{q}, J=7.3 \mathrm{~Hz}, 2 \mathrm{H}, \mathrm{CH}{ }_{2} \mathrm{OH}\right)$, 1.66-1.54 (m, 2H, CH( $\left.\left(\mathrm{CH}_{3}\right)_{2}\right), 1.48-1.40\left(\mathrm{~m}, 2 \mathrm{H}, \mathrm{CHCH}_{2(\mathrm{a})}\right), 1.38-1.33\left(\mathrm{~m}, 2 \mathrm{H}, \mathrm{CHCH}_{2(\mathrm{~b})}\right)$, $0.88\left(\mathrm{~d}, J=6.6 \mathrm{~Hz}, 6 \mathrm{H}, \mathrm{CH}\left(\mathrm{CH}_{3}\right)_{2}\right), 0.86\left(\mathrm{~d}, J=6.6 \mathrm{~Hz}, 6 \mathrm{H}, \mathrm{CH}\left(\mathrm{CH}_{3}\right)_{2}\right) .{ }^{13} \mathrm{C}-\mathrm{NMR}(126 \mathrm{MHz}$, DMSO- $\left.d_{6}\right): \delta(\mathrm{ppm})=160.5,143.4,128.0,63.8,49.7,45.4,24.4,23.3,21.9 ; \mathrm{LC} / \mathrm{MS}$ (ESI): found $371.2[\mathrm{M}+\mathrm{H}]^{+}, \mathrm{C}_{18} \mathrm{H}_{30} \mathrm{~N}_{2} \mathrm{O}_{4} \mathrm{~S}$ requires 370.19; anal. calcd. for $\mathrm{C}_{18} \mathrm{H}_{30} \mathrm{~N}_{2} \mathrm{O}_{4} \mathrm{~S}$ : C, 58.35; $\mathrm{H}$, 8.16; N, 7.56; found: C, 58.33; H, 8.18; N, 7.55.

\subsection{3. $N^{2}, N^{5}$-Bis((2S,3R)-1-Hydroxy-3-methylpentan-2-yl)thiophene-2,5-dicarboxamide (4c)}

Following GP1, thiophene-2,5-dicarboxlyic acid chloride (2) and (2S,3R)-2-amino-3methylpentan-1-ol (3c) reacted to produce 2,5-dicarboxamide alcohol (4c) as white solid $(1.04 \mathrm{~g}, 97 \%)$; m.p. $233-234^{\circ} \mathrm{C} ;[\alpha]_{\mathrm{D}}^{20}=-30.39^{\circ}\left(c 0.10, \mathrm{CH}_{3} \mathrm{OH}\right)$; IR $\left(\mathrm{KBr}, \mathrm{cm}^{-1}\right)$ : 3352, 3086, 2956, 2870, 2609, 2493, 1625, 1544, 1516, 1465, 1030, 744; ${ }^{1} \mathrm{H}-\mathrm{NMR}$ (500 MHz, DMSO-d ${ }_{6}$ ): $\delta(\mathrm{ppm})=8.09(\mathrm{~d}, J=8.9 \mathrm{~Hz}, 2 \mathrm{H}, \mathrm{NH}), 7.76(\mathrm{~s}, 2 \mathrm{H}, \mathrm{Ar}-\mathrm{H}), 4.53\left(\mathrm{~s}, 2 \mathrm{H}, \mathrm{CH}_{2} \mathrm{OH}\right), 3.78-3.70$ (m, 2H, NHCH), 3.53-3.42 (m, 4H, CH $\left.\mathrm{CH}_{2} \mathrm{OH}\right), 1.68-1.59\left(\mathrm{~m}, 2 \mathrm{H}, \mathrm{CHCH}_{3}\right), 1.47-1.37(\mathrm{~m}, 2 \mathrm{H}$, $\left.\mathrm{CH}_{2} \mathrm{CH}_{3}\right), 1.12-1.01\left(\mathrm{~m}, 2 \mathrm{H}, \mathrm{CH}_{2} \mathrm{CH}_{3}\right), 0.83\left(\mathrm{~d}, J=6.9 \mathrm{~Hz}, 6 \mathrm{H}, \mathrm{CHCH}_{3}\right), 0.80(\mathrm{t}, J=7.4 \mathrm{~Hz}$, $\left.6 \mathrm{H}, \mathrm{CH}_{2} \mathrm{CH}_{3}\right) ;{ }^{13} \mathrm{C}-\mathrm{NMR}\left(126 \mathrm{MHz}, \mathrm{DMSO}-d_{6}\right): \delta(\mathrm{ppm})=160.7,143.4,128.0,60.9,55.7,35.1$, 25.1, 15.5, 11.2; LC/MS (ESI): found 399.3 [M + H] $]^{+}, \mathrm{C}_{20} \mathrm{H}_{34} \mathrm{~N}_{2} \mathrm{O}_{4} \mathrm{~S}$ requires 398.22; anal. calcd. for $\mathrm{C}_{18} \mathrm{H}_{30} \mathrm{~N}_{2} \mathrm{O}_{4} \mathrm{~S}$ : C, 58.35; H, 8.16; N, 7.56; found: C, 58.17; H, 8.26; N, 7.44.

\subsection{General Procedure (GP2) for the Preparation of Thiophene-2,5-bis-imidazoline Chiral Ligands (L1-L3)}

GP2: Thiophene-2,5-dicarboxamide alcohol (4a) $(1.0 \mathrm{~g}, 2.92 \mathrm{mmol})$ in $\mathrm{SOCl}_{2}(8.76 \mathrm{~mL})$ was refluxed for $24 \mathrm{~h}$. After removal of $\mathrm{SOCl}_{2}$, ice-water was added to the residue and the product was extracted with $\mathrm{CH}_{2} \mathrm{Cl}_{2}(3 \times 25 \mathrm{~mL})$. The combined extracts were washed with brine and dried over anhydrous $\mathrm{Na}_{2} \mathrm{SO}_{4}$. The organics were evaporated to give the crude thiophene-2,5-dicarboxamid dichloride (5a). The crude dichloride (5a) was then dissolved in dry diethyl ether $(20 \mathrm{~mL})$ and the insoluble impurities were filtered out. To this solution, dry triethylamine $(4.9 \mathrm{~mL}, 35.0 \mathrm{mmol}, 12.0$ eq.) was added, followed by arylamine $(6 \mathbf{a}-\mathbf{c})(2.5$ eq. $)$. After stirring for $12 \mathrm{~h}$ at room temperature, $15 \% \mathrm{NaOH}(15 \mathrm{~mL})$ was added and stirred for another $24 \mathrm{~h}$. The aqueous portion was extracted with dichloromethane $(3 \times 20 \mathrm{~mL})$ and then washed with brine. The combined organics were dried over anhydrous $\mathrm{Na}_{2} \mathrm{SO}_{4}$ and concentrated under reduced pressure to afford crude thiophene-2,5bis(imidazolinyl)thiophene ligands (L1-L3). The pure ligands (L1-L3) were isolated by column chromatography, using the combination of ethylacetate/petroleumether $/ \mathrm{Et}_{3} \mathrm{~N}$ $(v: v: v=75: 24: 1)$ as an eluent.

\subsubsection{2,5-Bis((S)-4-IsoPropyl-1-phenyl-4,5-dihydro-1H-imidazol-2-yl)thiophene (L1)}

Thiophene-2,5-dicarboxamide alcohol $4 \mathbf{a}(1.0 \mathrm{~g}, 2.92 \mathrm{mmol})$ and aniline $6 \mathbf{6}(0.68 \mathrm{~g}, 7.3$ $\mathrm{mmol}$ ) were reacted according to GP2 and afforded yellow-colored ligand L1 (yield $533 \mathrm{mg}$, $40 \%) ;[\alpha]_{\mathrm{D}}^{20}=+86.24^{\circ}\left(c\right.$ 0.106, EtOH) ${ }^{1} \mathrm{H}-\mathrm{NMR}\left(400 \mathrm{MHz}, \mathrm{DMSO}-d_{6}\right): \delta(\mathrm{ppm})=7.27(\mathrm{t}$, $J=7.7 \mathrm{~Hz}, 4 \mathrm{H}, \mathrm{Ar}-\mathrm{H}), 7.10(\mathrm{t}, J=7.3 \mathrm{~Hz}, 2 \mathrm{H}, \mathrm{Ar}-\mathrm{H}), 6.97$ (d, $J=8.1 \mathrm{~Hz}, 4 \mathrm{H}, \mathrm{Ar}-\mathrm{H}), 6.55$ (s, 2H, Ar-H), 4.00-3.86 (m, 4H, $\left.\mathrm{NCH}_{2}\right), 3.51(\mathrm{t}, J=8.1 \mathrm{~Hz}, 2 \mathrm{H}, \mathrm{NCH}), 1.72(\mathrm{p}, J=6.6 \mathrm{~Hz}, 2 \mathrm{H}$, $\left.\mathrm{CHCH}_{3}\right), 0.94\left(\mathrm{~d}, J=7.3 \mathrm{~Hz}, 6 \mathrm{H}, \mathrm{CHCH}_{3(\mathrm{a})}\right), 0.86\left(\mathrm{~d}, J=7.3 \mathrm{~Hz}, 6 \mathrm{H}, \mathrm{CHCH}_{3(\mathrm{~b})}\right) ;{ }^{13} \mathrm{C}-\mathrm{NMR}$ $\left(101 \mathrm{MHz}, \mathrm{DMSO}-d_{6}\right): \delta(\mathrm{ppm})=154.5,143.1,135.2,129.1,128.6124 .7,124.2,70.13,59.8,57.5$, 
32.7, 18.6; LC/MS (ESI): found 457.2 [M + H] $]^{+}, \mathrm{C}_{28} \mathrm{H}_{32} \mathrm{~N}_{4} \mathrm{~S}$ requires 456.65; anal. calcd. for $\mathrm{C}_{28} \mathrm{H}_{32} \mathrm{~N}_{4} \mathrm{~S}$ : C, 73.65; H, 7.06; N, 12.27; found: $\mathrm{C}, 73.60 ; \mathrm{H}, 7.04 ; \mathrm{N}, 12.25$.

3.3.2. 2,5-Bis((S)-1-(4-Chlorophenyl)-4-isopropyl-4,5-dihydro-1H-imidazol-2-yl)thiophene (L2)

Thiophene-2,5-dicarboxamide alcohol (4a) $(1.0 \mathrm{~g}, 2.92 \mathrm{mmol})$ and 4-chloroaniline (6b) $(0.93 \mathrm{~g}, 7.3 \mathrm{mmol})$ were reacted according to GP2 and afforded yellow-colored ligand L2 (yield $583 \mathrm{mg}, 38 \%) ;[\alpha]_{\mathrm{D}}^{20}=-153.29^{\circ}\left(c 0.07, \mathrm{CH}_{2} \mathrm{Cl}_{2}\right) ;{ }^{1} \mathrm{H}-\mathrm{NMR}\left(400 \mathrm{MHz}\right.$, DMSO- $\left.d_{6}\right)$ : $\delta(\mathrm{ppm})=7.32(\mathrm{~d}, J=8.8 \mathrm{~Hz}, 4 \mathrm{H}, \mathrm{Ar}-\mathrm{H}), 6.97(\mathrm{~d}, J=8.8 \mathrm{~Hz}, 4 \mathrm{H}, \mathrm{Ar}-\mathrm{H}), 6.68(\mathrm{~s}, 2 \mathrm{H}, \mathrm{Ar}-\mathrm{H})$, 4.00-3.90 (m, 4H, NCH $)_{2}, 3.54(\mathrm{t}, J=7.3 \mathrm{~Hz}, 2 \mathrm{H}, \mathrm{NCH}), 1.73\left(\mathrm{~h}, J=6.6 \mathrm{~Hz}, 2 \mathrm{H}, \mathrm{CHCH}_{3}\right)$, $0.93\left(\mathrm{~d}, J=6.6 \mathrm{~Hz}, 6 \mathrm{H}, \mathrm{CHCH}_{3(\mathrm{a})}\right), 0.85\left(\mathrm{~d}, J=6.6 \mathrm{~Hz}, 6 \mathrm{H}, \mathrm{CHCH}_{3(\mathrm{~b})}\right) ;{ }^{13} \mathrm{C} \mathrm{NMR}(101 \mathrm{MHz}$, DMSO- $\left.d_{6}\right) \delta(\mathrm{ppm})=154.0,141.7,134.8,129.1,128.6,125.5,125.3,70.0,57.1,54.9,32.6,18.7$; LC/MS (ESI): found 525.2 [M + H] $]^{+}$, for $\mathrm{C}_{28} \mathrm{H}_{30} \mathrm{Cl}_{2} \mathrm{~N}_{4} \mathrm{~S}$ requires 524.16; anal. calcd. for $\mathrm{C}_{28} \mathrm{H}_{30} \mathrm{Cl}_{2} \mathrm{~N}_{4} \mathrm{~S}: \mathrm{C}, 63.99 ; \mathrm{H}, 5.75 ; \mathrm{N}, 10.66$; found: $\mathrm{C}, 63.87 ; \mathrm{H}, 5.72 ; \mathrm{N}, 10.61$.

\subsubsection{2,5-Bis((S)-4-IsoPropyl-1-( $p$-tolyl)-4,5-dihydro-1H-imidazol-2-yl)thiophene (L3)}

Thiophene-2,5-dicarboxamide alcohol (4a) $(1.0 \mathrm{~g}, 2.92 \mathrm{mmol})$ and $p$-toluidine $6 \mathbf{c}$ $(0.78 \mathrm{~g}, 7.3 \mathrm{mmol})$ were reacted according to GP2 and afforded yellow-colored ligand L3 (yield $538 \mathrm{mg}, 38 \%) ;[\alpha]_{\mathrm{D}}^{20}=+92.53^{\circ}(c 0.05, \mathrm{EtOH}) ;{ }^{1} \mathrm{H}-\mathrm{NMR}\left(400 \mathrm{MHz}, \mathrm{DMSO}-d_{6}\right)$ : $\delta(\mathrm{ppm})=7.08(\mathrm{~d}, J=8.1 \mathrm{~Hz}, 4 \mathrm{H}, \mathrm{Ar}-\mathrm{H}), 6.89(\mathrm{~d}, J=8.1 \mathrm{~Hz}, 4 \mathrm{H}, \mathrm{Ar}-\mathrm{H}), 6.52(\mathrm{~s}, 2 \mathrm{H}, \mathrm{Ar}-\mathrm{H})$, 3.93-3.86 (m, 4H, NCH $), 3.47-3.41(\mathrm{~m}, 2 \mathrm{H}, \mathrm{NCH}), 2.24\left(\mathrm{~s}, 6 \mathrm{H}, \mathrm{PhCH}_{3}\right), 1.73(\mathrm{q}, J=6.6 \mathrm{~Hz}$, $\left.2 \mathrm{H}, \mathrm{CHCH}_{3}\right), 0.94\left(\mathrm{~d}, J=7.3 \mathrm{~Hz}, 6 \mathrm{H}, \mathrm{CHCH}_{3(\mathrm{a})}\right), 0.85\left(\mathrm{~d}, J=6.6 \mathrm{~Hz}, 6 \mathrm{H}, \mathrm{CHCH}_{3(\mathrm{~b})}\right) ;{ }^{13} \mathrm{C}$ NMR (101 MHz, DMSO- $d_{6}$ ) $\delta(\mathrm{ppm}) 154.8,140.7,135.2,134.3,129.6,128.6,124.5,70.1,57.8$, 32.8, 20.5, 18.7, 18.1; LC/MS (ESI): found $485.3[\mathrm{M}+\mathrm{H}]^{+}$, for $\mathrm{C}_{30} \mathrm{H}_{36} \mathrm{~N}_{4} \mathrm{~S}$ requires 484.27; anal. calcd. for $\mathrm{C}_{30} \mathrm{H}_{36} \mathrm{~N}_{4} \mathrm{~S}$ : C, 74.34; $\mathrm{H}, 7.49 ; \mathrm{N}, 11.56$; found: $\mathrm{C}, 74.30 ; \mathrm{H}, 7.48 ; \mathrm{N}, 11.52$.

\subsection{General Procedure (GP3) for the Synthesis of Thiophene-2,5-bis-oxazoline Chiral Ligands (L4 and L5)}

GP3: Thiophene-2,5-dicarboxamide alcohol $(4 \mathbf{b}-\mathbf{c})(2.92 \mathrm{mmol})$ was added to the solution of $\mathrm{CH}_{2} \mathrm{Cl}_{2}(60 \mathrm{~mL})$ and triethylamine ( 4.0 eq., $\left.1.18 \mathrm{~g}, 11.7 \mathrm{mmol}\right)$. Catalytic amounts of DMAP (36 mg, 0.1 eq.) and $p$-tosylchoride ( $695 \mathrm{mg}, 3.65 \mathrm{mmol}, 1.25$ eq.) were added, and the mixture was stirred at $0{ }^{\circ} \mathrm{C}$ to r.t. for $48 \mathrm{~h}$. After completion of the reaction, saturated aqueous ammonium chloride solution $(100 \mathrm{~mL})$ was added and stirred for another $10 \mathrm{~min}$ at room temperature. The organic layer was extracted with $\mathrm{CH}_{2} \mathrm{Cl}_{2}(3 \times 25 \mathrm{~mL})$ and washed with saturated aqueous $\mathrm{NaHCO}_{3}$ solution $(50 \mathrm{~mL})$. The combined organic layers were dried over anhydrous $\mathrm{Na}_{2} \mathrm{SO}_{4}$, and the solvent was evaporated in vacuum to afford crude ligands ( $\mathbf{L} 4$ and $\mathbf{L 5}$ ), which was purified by column chromatography $\left(5 \% \mathrm{CH}_{2} \mathrm{Cl}_{2} / \mathrm{CH}_{3} \mathrm{OH}\right)$ to afford pure thiophene-2,5-bis(oxazolinyl)thiophene ligands (L4 and L5).

\subsubsection{2,5-Bis((S)-4-isoButyl-4,5-dihydrooxazol-2-yl)thiophene (L4)}

Following the GP2, thiophene-2,5-dicarboxamide (4b) (1.08 g, $2.92 \mathrm{mmol})$ underwent direct ring closure reaction to afford ligand $\mathbf{L 4}$ as white solid (yield $586 \mathrm{mg}, 60 \%$ ); m.p. $48-50^{\circ} \mathrm{C} ;[\alpha]_{\mathrm{D}}^{20}=-46.88^{\circ}\left(c 0.093, \mathrm{CH}_{3} \mathrm{OH}\right) ; \mathrm{IR}\left(\mathrm{KBr}, \mathrm{cm}^{-1}\right): 3104,2953,2920,2870,2847$, $1647,1533,1251,1051,1019,944,829 ;{ }^{1} \mathrm{H}-\mathrm{NMR}\left(500 \mathrm{MHz}, \mathrm{DMSO}-d_{6}\right): \delta(\mathrm{ppm})=7.53(\mathrm{~s}, 2 \mathrm{H}$, Ar-H), $4.54\left(\mathrm{dd}, J=9.3,8.0 \mathrm{~Hz}, 2 \mathrm{H}, \mathrm{OCH}_{2(\mathrm{a})}\right), 4.31-4.23(\mathrm{~m}, 2 \mathrm{H}, \mathrm{NCH}), 3.97(\mathrm{t}, J=8.2 \mathrm{~Hz}$, $\left.2 \mathrm{H}, \mathrm{OCH}_{2(\mathrm{~b})}\right), 1.76\left(\mathrm{dt}, J=13.5,6.7 \mathrm{~Hz}, 2 \mathrm{H}, \mathrm{CH}\left(\mathrm{CH}_{3}\right)_{2}\right), 1.52(\mathrm{dt}, J=13.9,7.0 \mathrm{~Hz}, 2 \mathrm{H}$, $\left.\mathrm{CHCH}_{2(\mathrm{a})}\right), 1.35\left(\mathrm{dt}, J=13.5,7.2 \mathrm{~Hz}, 2 \mathrm{H}, \mathrm{CHCH}_{2(\mathrm{~b})}\right), 0.93\left(\mathrm{~d}, J=3.9 \mathrm{~Hz}, 6 \mathrm{H}, \mathrm{CH}\left(\mathrm{CH}_{3}\right)_{2}\right)$, $0.91\left(\mathrm{~d}, J=3.7 \mathrm{~Hz}, 6 \mathrm{H}, \mathrm{CH}\left(\mathrm{CH}_{3}\right)_{2}\right) ;{ }^{13} \mathrm{C}-\mathrm{NMR}\left(126 \mathrm{MHz}, \mathrm{DMSO}-d_{6}\right): \delta(\mathrm{ppm})=157.1,133.3$, 130.5, 73.3, 64.9, 44.7, 25.0, 22.7, 22.5; LC/MS (ESI): found $335.2[\mathrm{M}+\mathrm{H}]^{+}, \mathrm{C}_{18} \mathrm{H}_{26} \mathrm{~N}_{2} \mathrm{O}_{2} \mathrm{~S}$ requires 334.17; anal. calcd. for $\mathrm{C}_{18} \mathrm{H}_{26} \mathrm{~N}_{2} \mathrm{O}_{2} \mathrm{~S}: \mathrm{C}, 64.64 ; \mathrm{H}, 7.84 ; \mathrm{N}, 8.38$; found: $\mathrm{C}, 64.62 ; \mathrm{H}$, $7.86 ; \mathrm{N}, 8.34$.

\subsubsection{2,5-Bis((S)-4-((S)-sec-Butyl)-4,5-dihydrooxazol-2-yl)thiophene (L5)}

Following the GP2, thiophene-2,5-dicarboxamide (4c) (1.08 g, $2.92 \mathrm{mmol})$ underwent direct ring closure reaction to afford ligand $\mathbf{L} 5$ as white solid (yield $537 \mathrm{mg}, 55 \%$ ); m.p.: 
$42-43^{\circ} \mathrm{C} ;[\alpha]_{\mathrm{D}}^{20}=-4.06^{\circ}\left(c 0.081, \mathrm{CH}_{3} \mathrm{OH}\right) ; \mathrm{IR}\left(\mathrm{KBr}, \mathrm{cm}^{-1}\right): 3102,2954,2921,2870,2845$, $1648,1533,1251,1052,1019,942,826 ;{ }^{1} \mathrm{H}-\mathrm{NMR}\left(500 \mathrm{MHz}, \mathrm{DMSO}-d_{6}\right): \delta(\mathrm{ppm})=7.53(\mathrm{~s}$, $2 \mathrm{H}, \mathrm{Ar}-\mathrm{H}), 4.47-4.41(\mathrm{~m}, 2 \mathrm{H}, \mathrm{NCH}), 4.18-4.11\left(\mathrm{~m}, 4 \mathrm{H}, \mathrm{OCH}_{2}\right), 1.62-1.50\left(\mathrm{~m}, 4 \mathrm{H}, \mathrm{CH}_{2} \mathrm{CH}_{3}\right)$,

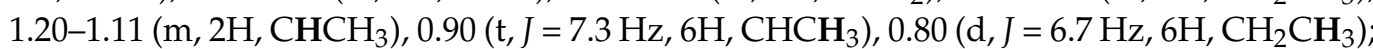
${ }^{13} \mathrm{C}-\mathrm{NMR}\left(126 \mathrm{MHz}, \mathrm{DMSO}-d_{6}\right): \delta(\mathrm{ppm})=157.2,133.2,130.4,70.9,70.2,38.6,25.4,14.4$, 11.3; LC/MS (ESI): found 335.2 [M + H] $]^{+}, \mathrm{C}_{18} \mathrm{H}_{26} \mathrm{~N}_{2} \mathrm{O}_{2} \mathrm{~S}$ requires 334.17; anal. calcd. for $\mathrm{C}_{18} \mathrm{H}_{26} \mathrm{~N}_{2} \mathrm{O}_{2} \mathrm{~S}: \mathrm{C}, 64.64 ; \mathrm{H}, 7.84 ; \mathrm{N}, 8.38$; found: $\mathrm{C}, 64.60 ; \mathrm{H}, 7.84 ; \mathrm{N}, 8.38$.

\subsection{Synthesis of the $\beta$-nitrostyrene ( $9 a-j)$}

All the $\beta$-nitrostyrenes $(\mathbf{9 a}-\mathbf{j})$ were synthesized by using well-known methods reported in the literature [84]. An oven-dried round bottom flask $(100 \mathrm{~mL})$ was charged with aldehydes $(10.0 \mathrm{mmol})$, nitromethane $(3.70 \mathrm{~g}, 60.0 \mathrm{mmol})$, piperidine $(85 \mathrm{mg}, 1.0 \mathrm{mmol})$ and toluene as solvent $(10 \mathrm{~mL})$. Anhydrous $\mathrm{FeCl}_{3}(16.2 \mathrm{mg}, 1.0 \mathrm{mmol})$ was then added to it. The reaction mixture was reflux gently for $4 \mathrm{~h}$ under dry condition, using guard tube. The completion of the reaction was confirmed by TLC, and the reaction mixture was cooled to room temperature. The excess solvent was removed under reduced pressure, and the residue was purified by silica gel (100-200 mesh) column chromatography to afford pure $\beta$-nitrostyrenes $\mathbf{9 a}-\mathbf{j}$ as yellow solid product (yield $75-90 \%$ ).

\subsection{Synthesis of Racemic Friedal-Crafts Alkylated Product Race-(10a-t)}

The racemic products were synthesized by using the reported method $[41,83]$. Indole derivatives $(0.30 \mathrm{mmol}), \beta$-nitrostyrenes $9 \mathbf{a}-\mathbf{h}(0.30 \mathrm{mmol}), \mathrm{FeCl}_{3}(10 \mathrm{~mol} \%)$ and $\mathrm{H}_{2} \mathrm{O}(2 \mathrm{~mL})$ were heated at $80{ }^{\circ} \mathrm{C}$ for the appropriate time $(24 \mathrm{~h})$. After the completion of the reaction, monitored by thin-layer chromatography (TLC), the product was extracted with ethyl acetate $(2 \times 20 \mathrm{~mL})$. The combined organic layer was dried over anhydrous sodium sulfate, evaporated under reduced pressure and purified by silica gel (100-200 mesh) column chromatography using $15 \%$ ethylacetate/ $n$-hexane as eluent to afford the pure racemic Friedal-Crafts alkylated product race-(10a-t) (yield $85-90 \%$ ).

\subsection{General Procedure (GP4) for the Asymmetric Friedal-Crafts Alkylation of Indole to $\beta$-nitrostyrene (10a-t)}

GP4: An oven-dried screw-capped vial $(8 \mathrm{~mL})$ was charged with ligand $\mathbf{L} 5(10 \mathrm{mg}$, $0.03 \mathrm{mmol}, 15 \mathrm{~mol} \%), \mathrm{Cu}(\mathrm{OTf})_{2}(11 \mathrm{mg}, 0.03 \mathrm{mmol}, 15 \mathrm{~mol} \%)$ and dry toluene $(3 \mathrm{~mL})$. The mixture was then stirred at reflux for $2 \mathrm{~h}$. After cooling to room temperature, $\beta$-nitrostyrene 9a-h $(0.2 \mathrm{mmol})$ and $4 \mathrm{~A}^{\circ}$ molecular sieves were added. Then, the mixture was stirred for another $30 \mathrm{~min}$, followed by addition of indole $8 \mathbf{a}-\mathbf{d}(0.2 \mathrm{mmol})$. The reaction was then left stirring for $48 \mathrm{~h}$ at room temperature. The solvent was removed under reduced pressure, and the crude product was isolated by flash column chromatography on silica gel with ethylacetate $/ n$-hexane $(2: 8, v / v)$ as eluent to afford pure Friedel-Crafts product $(\mathbf{1 0 a}-\mathbf{t})$ in $35-76 \%$ isolated yield with $21-81 \%$ enantiomeric excess (ee).

\subsection{1. (S)-3-(1-(4-Fluorophenyl)-2-nitroethyl)-1H-indole (10a)}

Indole $8 \mathrm{a}(24 \mathrm{mg}, 0.2 \mathrm{mmol})$ and 4-floronitrostyrene $(9 \mathrm{a})(34 \mathrm{mg}, 0.2 \mathrm{mmol})$ were reacted according to the GP4 to yield product 10a as colorless oil (isolated yield $38 \mathrm{mg}$, $67 \%$ ). Enantiomeric excess (ee) was determined by chiral HPLC (Chiracel OD-H column) $\left(70 \% n\right.$-hexane $\left./ i-\mathrm{PrOH}, 1.0 \mathrm{~mL} / \mathrm{min} ; t_{\text {major }}=24.85 \mathrm{~min} ; t_{\text {minor }}=30.28 \mathrm{~min} ; \lambda=254 \mathrm{~nm}\right)$; $74.3 \% e e ;[\alpha]_{\mathrm{D}}^{20}=+32.98^{\circ}\left(c 0.10, \mathrm{CH}_{3} \mathrm{OH}\right) ;\left[\right.$ Lit. [74] $[\alpha]_{\mathrm{D}}^{20}=+39.9^{\circ}\left(c 0.85, \mathrm{CH}_{2} \mathrm{Cl}_{2}\right)$; ${ }^{1} \mathrm{H}-\mathrm{NMR}\left(500 \mathrm{MHz}, \mathrm{CDCl}_{3}\right): \delta(\mathrm{ppm})=8.13(\mathrm{~s}, 1 \mathrm{H}, \mathrm{NH}), 7.47-7.40(\mathrm{~m}, 1 \mathrm{H}, \mathrm{Ar}-\mathrm{H}), 7.35(\mathrm{~s}$, $1 \mathrm{H}, \mathrm{Ar}-\mathbf{H}), 7.32-7.28(\mathrm{~m}, 2 \mathrm{H}, \mathrm{Ar}-\mathbf{H}), 7.23$ (ddd, $J=8.2,7.0,1.2 \mathrm{~Hz}, 1 \mathrm{H}, \mathrm{Ar}-\mathbf{H}), 7.11$ (ddd, $J=8.1,6.9,1.0 \mathrm{~Hz}, 1 \mathrm{H}, \mathrm{Ar}-\mathbf{H}), 7.04-6.96(\mathrm{~m}, 3 \mathrm{H}, \mathrm{Ar}-\mathbf{H}), 5.19(\mathrm{t}, J=8.0 \mathrm{~Hz}, 1 \mathrm{H}, \mathrm{CH}), 5.05$ $\left(\mathrm{dd}, J=12.5,7.5 \mathrm{~Hz}, 1 \mathrm{H}, \mathrm{CH}_{2(\mathrm{a})}\right), 4.90\left(\mathrm{dd}, J=12.5,8.6 \mathrm{~Hz}, 1 \mathrm{H}, \mathrm{CH}_{2(\mathrm{~b})}\right) ;{ }^{13} \mathrm{C}-\mathrm{NMR}(126 \mathrm{MHz}$, $\left.\mathrm{CDCl}_{3}\right): \delta(\mathrm{ppm})=163.1$ and $161.18\left(\mathrm{C}_{1}-\mathrm{F}, J_{\mathrm{C}-\mathrm{F}}=246.58 \mathrm{~Hz}\right), 136.6,135.07$ and $135.04\left(\mathrm{C}_{4}-\mathrm{F}\right.$, $\left.J_{\mathrm{C}-\mathrm{F}}=3.15 \mathrm{~Hz}\right), 129.50$ and $129.44\left(\mathrm{C}_{3}-\mathrm{F}, J_{\mathrm{C}-\mathrm{F}}=7.94 \mathrm{~Hz}\right), 126.0,122.9,121.6,120.1,118.9$, 
115.99 and $115.82\left(\mathrm{C}_{2}-\mathrm{F}, J_{\mathrm{C}-\mathrm{F}}=21.67 \mathrm{~Hz}\right), 114.2,111.6,79.6,41.0$. All the analytical data are in accordance with the reported literature $[42,74]$.

\subsection{2. (S)-3-(1-(3-Bromophenyl)-2-nitroethyl)-1H-indole (10b)}

Indole $8 \mathbf{a}(24 \mathrm{mg}, 0.2 \mathrm{mmol})$ and 3-bromonitrostyrene $(9 \mathrm{~b})(46 \mathrm{mg}, 0.2 \mathrm{mmol})$ were reacted according to the GP4 to yield product $10 \mathrm{~b}$ as colorless oil (isolated yield $44 \mathrm{mg}$, $64 \%$ ). Enantiomeric excess (ee) was determined by chiral HPLC (Chiracel OD-H column) $\left(70 \% n\right.$-hexane $/ i$-PrOH, $\left.1.0 \mathrm{~mL} / \mathrm{min} ; t_{\text {major }}=27.66 \mathrm{~min} ; t_{\operatorname{minor}}=36.16 \mathrm{~min} ; \lambda=254 \mathrm{~nm}\right)$; $79.5 \%$ ee; $[\alpha]_{\mathrm{D}}^{20}=+14.41^{\circ}\left(c 0.104, \mathrm{CH}_{3} \mathrm{OH}\right)$; [Lit. [74] $[\alpha]_{\mathrm{D}}^{20}=+14.7^{\circ}\left(c 1.3, \mathrm{CH}_{2} \mathrm{Cl}_{2}\right] ;{ }^{1} \mathrm{H}-$ $\mathrm{NMR}\left(500 \mathrm{MHz}, \mathrm{CDCl}_{3}\right): \delta(\mathrm{ppm})=8.12(\mathrm{~s}, 1 \mathrm{H}, \mathrm{NH}), 7.49-7.32(\mathrm{~m}, 4 \mathrm{H}, \mathrm{Ar}-\mathrm{H}), 7.28-7.08$ $(\mathrm{m}, 4 \mathrm{H}, \mathrm{Ar}-\mathrm{H}), 6.98$ (d, J = 2.5 Hz, 1H, Ar-H), 5.15 (t, $J=8.0 \mathrm{~Hz}, 1 \mathrm{H}, \mathrm{CH}), 5.02$ (dd, J = 12.8, $\left.7.6 \mathrm{~Hz}, 1 \mathrm{H}, \mathrm{CH}_{2(\mathrm{a})}\right), 4.89$ (dd, $\left.J=12.0,7.8 \mathrm{~Hz}, 1 \mathrm{H}, \mathrm{CH}_{2(\mathrm{~b})}\right) ;{ }^{13} \mathrm{C}-\mathrm{NMR}\left(126 \mathrm{MHz} \mathrm{CDCl}_{3}\right)$ : $\delta(\mathrm{ppm})=141.7,136.5,130.9,130.9,130.6,126.6,126.0,123.1,122.9,121.7,120.2,118.8,113.6$, 111.6, 79.2, 41.2. All the analytical data are in accordance with the reported literature $[74,76]$.

\subsection{3. (S)-3-(2-Nitro-1-(4-(trifluoromethyl)phenyl)ethyl)-1H-indole (10c)}

Indole $8 \mathrm{a}$ (24 mg, $0.2 \mathrm{mmol})$ and 4-trifluoromethynitrostyrene 9c (44 mg, $0.2 \mathrm{mmol})$ were reacted according to the GP4 to yield product 10c as colorless oil (isolated yield $27 \mathrm{mg}$, $40 \%$ ). Enantiomeric excess (ee) was determined by chiral HPLC (Chiracel OD-H column) $\left(70 \%\right.$-hexane $/ i$-PrOH, $\left.1.0 \mathrm{~mL} / \mathrm{min} ; t_{\text {major }}=32.09 \mathrm{~min} ; t_{\text {minor }}=39.93 \mathrm{~min} ; \lambda=254 \mathrm{~nm}\right)$; $75.4 \%$ ee; $[\alpha]_{\mathrm{D}}^{20}=+6.93^{\circ}\left(c 0.05, \mathrm{CH}_{3} \mathrm{OH}\right)$; [Lit. [75] $[\alpha]_{\mathrm{D}}^{20}=+2.9^{\circ}$ (c 1.0, $\left.\mathrm{CHCl}_{3}\right]$; ${ }^{1} \mathrm{H}-\mathrm{NMR}$ $\left(500 \mathrm{MHz}, \mathrm{CDCl}_{3}\right): \delta(\mathrm{ppm})=8.17(\mathrm{~s}, 1 \mathrm{H}, \mathrm{NH}), 7.59(\mathrm{~d}, J=8.1 \mathrm{~Hz}, 2 \mathrm{H}, \mathrm{Ar}-\mathrm{H}), 7.47(\mathrm{~d}$, $J=8.1 \mathrm{~Hz}, 2 \mathrm{H}, \mathrm{Ar}-\mathrm{H}), 7.42(\mathrm{dq}, J=8.0,1.0 \mathrm{~Hz}, 1 \mathrm{H}, \mathrm{Ar}-\mathrm{H}), 7.38(\mathrm{dt}, J=8.3,0.9 \mathrm{~Hz}, 1 \mathrm{H}$, Ar-H), $7.23(\mathrm{ddd}, J=8.2,7.1,1.2 \mathrm{~Hz}, 1 \mathrm{H}, \mathrm{Ar}-\mathbf{H}), 7.13-7.08(\mathrm{~m}, 1 \mathrm{H}, \mathrm{Ar}-\mathbf{H}), 7.03$ (dd, $J=2.6$, $0.9 \mathrm{~Hz}, 1 \mathrm{H}, \mathrm{Ar}-\mathbf{H}), 5.26(\mathrm{t}, J=8.0 \mathrm{~Hz}, 1 \mathrm{H}, \mathrm{CH}), 5.09\left(\mathrm{dd}, J=12.8,7.4 \mathrm{~Hz}, 1 \mathrm{H}, \mathrm{CH}_{2(\mathrm{a})}\right)$, $4.97\left(\mathrm{dd}, J=12.7,8.7 \mathrm{~Hz}, 1 \mathrm{H}, \mathrm{CH}_{2(\mathrm{~b})}\right) ;{ }^{13} \mathrm{C}-\mathrm{NMR}\left(126 \mathrm{MHz} \mathrm{CDCl}_{3}\right): \delta(\mathrm{ppm})=143.4,136.6$, $128.3,126.11,126.07,126.04,125.9,123.1,121.8,120.3,118.8,113.6,111.7,79.1,41.4$. All the analytical data are in accordance with the reported literature [75].

\subsection{4. (S)-3-(1-(4-Methoxyphenyl)-2-nitroethyl)-1H-indole (10d)}

Indole 8a (24 mg, $0.2 \mathrm{mmol})$ and 4-methoxynitrostyrene $9 \mathrm{~d}$ (36 $\mathrm{mg}, 0.2 \mathrm{mmol})$ were reacted according to the GP4 to yield product $10 \mathrm{~d}$ as white solid (isolated yield $39 \mathrm{mg}$, $66 \%$ ), m.p. $148-149{ }^{\circ} \mathrm{C}$; Enantiomeric excess (ee) was determined by chiral HPLC (Chiracel OD-H column) $\left(70 \% n\right.$-hexane $/ i$-PrOH, $1.0 \mathrm{~mL} / \mathrm{min} ; t_{\mathrm{major}}=26.24 \mathrm{~min} ; t_{\mathrm{minor}}=32.20 \mathrm{~min}$; $\lambda=254 \mathrm{~nm}) ; 69.3 \%$ ee; $[\alpha]_{\mathrm{D}}^{20}=+12.13^{\circ}\left(c 0.53, \mathrm{CH}_{3} \mathrm{OH}\right)$ [Lit.[74] $[\alpha]_{\mathrm{D}}^{20}=+26.4^{\mathrm{o}}($ c 1.1, $\left.\left.\mathrm{CH}_{2} \mathrm{Cl}_{2}\right)\right] ;{ }^{1} \mathrm{H}-\mathrm{NMR}\left(500 \mathrm{MHz} \mathrm{CDCl}_{3}\right): \delta(\mathrm{ppm})=8.09(\mathrm{~s}, 1 \mathrm{H}, \mathrm{NH}), 7.44(\mathrm{~d}, J=8.0 \mathrm{~Hz}, 1 \mathrm{H}$, Ar-H), $7.36(\mathrm{dd}, J=8.2,1.0 \mathrm{~Hz}, 1 \mathrm{H}, \mathrm{Ar}-\mathbf{H}), 7.29-7.23(\mathrm{~m}, 2 \mathrm{H}, \mathrm{Ar}-\mathbf{H}), 7.20(\mathrm{tt}, J=8.2,1.2 \mathrm{~Hz}$, 1H, Ar-H), 7.12-7.06 (m, 1H, Ar-H), $7.02(\mathrm{dd}, J=2.5,1.1 \mathrm{~Hz}, 1 \mathrm{H}, \mathrm{Ar}-\mathbf{H}), 6.91-6.81(\mathrm{~m}, 2 \mathrm{H}$, Ar-H), $5.14(\mathrm{t}, J=8.0 \mathrm{~Hz}, 1 \mathrm{H}, \mathrm{CH}), 5.05\left(\mathrm{dd}, J=12.4,7.5 \mathrm{~Hz}, 1 \mathrm{H}, \mathrm{CH}_{2(\mathrm{a})}\right), 4.90$ (dd, $J=12.4$, $\left.8.5 \mathrm{~Hz}, 1 \mathrm{H}, \mathrm{CH}_{2(\mathrm{~b})}\right), 3.78\left(\mathrm{~s}, 3 \mathrm{H}, \mathrm{CH}_{3}\right) ;{ }^{13} \mathrm{C}-\mathrm{NMR}\left(126 \mathrm{MHz} \mathrm{CDCl}_{3}\right): \delta(\mathrm{ppm})=159.0,136.6$, $131.3,129.0,126.2,122.8,121.6,120.1,119.1,114.9,114.4,111.5,79.9,55.4,41.0$. All the analytical data are in accordance with the reported literature $[42,74]$.

\subsection{5. (R)-3-(2-Nitro-1-(2-nitrophenyl)ethyl)-1H-indole (10e)}

Indole $8 \mathbf{a}(24 \mathrm{mg}, 0.2 \mathrm{mmol})$ and 2-nitronitrostyrene $9 \mathrm{e}(39 \mathrm{mg}, 0.2 \mathrm{mmol})$ were reacted according to the GP4 to yield product $10 \mathrm{~d}$ as yellow oil (isolated yield $36 \mathrm{mg}, 58 \%$ ). Enantiomeric excess (ee) was determined by chiral HPLC (Chiracel OD-H column) (70\% $n$-hexane $\left./ i-\mathrm{PrOH}, 1.0 \mathrm{~mL} / \mathrm{min} ; t_{\text {minor }}=37.48 \mathrm{~min} ; t_{\text {major }}=67.98 \mathrm{~min} ; \lambda=254 \mathrm{~nm}\right) ; 70.0 \%$ $e e ;[\alpha]_{\mathrm{D}}^{20}=+95.57^{\circ}\left(c 0.053, \mathrm{CH}_{3} \mathrm{OH}\right)$; [Lit. [80] $[\alpha]_{\mathrm{D}}^{20}=+55.3^{\circ}\left(\mathrm{c} 0.7, \mathrm{CH}_{2} \mathrm{Cl}_{2}\right)$ ]; ${ }^{1} \mathrm{H}-\mathrm{NMR}$ $\left(500 \mathrm{MHz}_{2} \mathrm{CDCl}_{3}\right): \delta(\mathrm{ppm})=8.23(\mathrm{~s}, 1 \mathrm{H}, \mathrm{NH}), 7.90(\mathrm{dd}, J=8.2,1.4 \mathrm{~Hz}, 1 \mathrm{H}, \mathrm{Ar}-\mathrm{H}), 7.48(\mathrm{td}$, $J=7.6,1.4 \mathrm{~Hz}, 1 \mathrm{H}, \mathrm{Ar}-\mathrm{H}), 7.43(\mathrm{dd}, J=7.9,1.6 \mathrm{~Hz}, 1 \mathrm{H}, \mathrm{Ar}-\mathrm{H}), 7.39(\mathrm{ddd}, J=8.5,7.3,1.6 \mathrm{~Hz}$, $1 \mathrm{H}, \mathrm{Ar}-\mathbf{H}), 7.35-7.30(\mathrm{~m}, 2 \mathrm{H}, \mathrm{Ar}-\mathbf{H}), 7.21-7.16(\mathrm{~m}, 1 \mathrm{H}, \mathrm{Ar}-\mathbf{H}), 7.12(\mathrm{~d}, J=2.6 \mathrm{~Hz}, 1 \mathrm{H}, \mathrm{Ar}-\mathbf{H})$, 7.07-7.02 (m, 1H, Ar-H), $5.88(\mathrm{t}, J=7.7 \mathrm{~Hz}, 1 \mathrm{H}, \mathrm{CH}), 5.12\left(\mathrm{dd}, J=13.2,7.1 \mathrm{~Hz}, 1 \mathrm{H}, \mathrm{CH}_{2(\mathrm{a})}\right)$, $5.07\left(\mathrm{dd}, J=13.2,8.3 \mathrm{~Hz}, 1 \mathrm{H}, \mathrm{CH}_{2(\mathrm{~b})}\right) ;{ }^{13} \mathrm{C}-\mathrm{NMR}\left(126 \mathrm{MHz}, \mathrm{CDCl}_{3}\right): \delta(\mathrm{ppm})=149.7,136.5$, 
$133.8,133.4,130.1,128.7,126.0,125.2,123.0,122.2,120.3,118.7,112.8,111.6,78.2,36.5$. All the analytical data are in accordance with the reported literature [80].

3.7.6. (R)-3-(1-(2,4-Dichlorophenyl)-2-nitroethyl)-1H-indole (10f)

Indole $8 \mathbf{a}(24 \mathrm{mg}, 0.2 \mathrm{mmol})$ and 2,4-dichloronitronitrostyrene $9 \mathrm{f}$ ( $44 \mathrm{mg}, 0.2 \mathrm{mmol})$ were reacted according to the GP4 to yield product $10 \mathrm{~d}$ as yellow oil (isolated yield $32 \mathrm{mg}$, $48 \%$ ). Enantiomeric excess (ee) was determined by chiral HPLC (Chiracel OD-H column) $\left(70 \% n\right.$-hexane $/ i$-PrOH, $\left.1.0 \mathrm{~mL} / \mathrm{min} ; t_{\text {minor }}=21.25 \mathrm{~min} ; t_{\text {major }}=35.78 \mathrm{~min} ; \lambda=254 \mathrm{~nm}\right)$; $71.25 \%$ ee; $[\alpha]_{\mathrm{D}}^{20}=+38.22^{\circ}\left(c 0.052, \mathrm{CH}_{3} \mathrm{OH}\right)$; [Lit. [74] $[\alpha]_{\mathrm{D}}^{20}=+59.5^{\circ}$ (c $\left.0.8, \mathrm{CH}_{2} \mathrm{Cl}_{2}\right)$ ]; ${ }^{1} \mathrm{H}-\mathrm{NMR}\left(500 \mathrm{MHz} \mathrm{CDCl}_{3}\right): \delta(\mathrm{ppm})=8.16(\mathrm{~s}, 1 \mathrm{H}, \mathrm{NH}), 7.47(\mathrm{t}, J=1.3 \mathrm{~Hz}, 1 \mathrm{H}, \mathrm{Ar}-\mathrm{H}), 7.38$ (ddt, $J=14.8,8.2,0.9 \mathrm{~Hz}, 2 \mathrm{H}, \mathrm{Ar}-\mathbf{H}), 7.24-7.20(\mathrm{~m}, 1 \mathrm{H}, \mathrm{Ar}-\mathbf{H}), 7.14(\mathrm{~d}, J=1.2 \mathrm{~Hz}, 2 \mathrm{H}, \mathrm{Ar}-\mathbf{H})$, 7.12-7.08 (m, 2H, Ar-H), 5.71-5.66 (m, 1H, CH), 4.99 (dd, J = 12.9, 8.7 Hz, 1H, CH 2(a) ), 4.93 $\left(\mathrm{dd}, J=12.9,7.0 \mathrm{~Hz}, 1 \mathrm{H}, \mathrm{CH}_{2(\mathrm{~b})}\right) ;{ }^{13} \mathrm{C}-\mathrm{NMR}\left(126 \mathrm{MHz}, \mathrm{CDCl}_{3}\right): \delta(\mathrm{ppm})=136.6,135.3,134.7$, $134.2,130.1,130.0,127.8,126.1,123.1,122.0,120.3,118.9,113.0,111.6,77.6,37.7$. All the analytical data are in accordance with the reported literature $[41,74]$.

\subsection{7. (S)-3-(2-Nitro-1-(thiophen-2-yl)ethyl)-1H-indole (10g)}

Indole $8 \mathbf{a}(24 \mathrm{mg}, 0.2 \mathrm{mmol})$ and (E)-2-(2-nitrovinyl)thiophene $9 \mathrm{~g}$ (31 $\mathrm{mg}, 0.2 \mathrm{mmol})$ were reacted according to the GP4 to yield product 10n as brown oil (isolated yield $28 \mathrm{mg}$, $52 \%$ ). Enantiomeric excess (ee) was determined by chiral HPLC (Chiracel OD-H column) $\left(75 \%\right.$-hexane $\left./ i-\mathrm{PrOH}, 1.0 \mathrm{~mL} / \mathrm{min} ; t_{\text {minor }}=28.57 \mathrm{~min} ; t_{\text {major }}=32.38 \mathrm{~min} ; \lambda=254 \mathrm{~nm}\right)$; $71.3 \%$ ee; $[\alpha]_{\mathrm{D}}^{20}=+32.18^{\circ}\left(c 0.037, \mathrm{CH}_{3} \mathrm{OH}\right) ;{ }^{1} \mathrm{H}-\mathrm{NMR}\left(500 \mathrm{MHz}, \mathrm{CDCl}_{3}\right): \delta(\mathrm{ppm})=8.15$ (s, 1H, NH), $7.53(\mathrm{~d}, J=8.0 \mathrm{~Hz}, 1 \mathrm{H}, \mathrm{Ar}-\mathrm{H}), 7.37(\mathrm{~d}, J=8.2 \mathrm{~Hz}, 1 \mathrm{H}, \mathrm{Ar}-\mathbf{H}), 7.25-7.21(\mathrm{~m}$, $1 \mathrm{H}, \mathrm{Ar}-\mathrm{H}), 7.20-7.18(\mathrm{~m}, 1 \mathrm{H}, \mathrm{Ar}-\mathbf{H}), 7.15-7.10(\mathrm{~m}, 1 \mathrm{H}, \mathrm{Ar}-\mathbf{H}), 7.09$ (d, J = 2.58 Hz, 1H, Ar-H), 7.01-6.99 (m, 1H, Ar-H), 6.95 (dd, J = 5.1, 3.6 Hz, 1H, Ar-H), 5.47 (t, J = 7.9 Hz, $1 \mathrm{H}, \mathrm{CH}), 5.08-4.96\left(\mathrm{~m}, 2 \mathrm{H}, \mathrm{CH}_{2}\right) ;{ }^{13} \mathrm{C}-\mathrm{NMR}\left(126 \mathrm{MHz}, \mathrm{CDCl}_{3}\right): \delta(\mathrm{ppm})=143.07,136.53$, $127.08,125.84,125.38,125.03,122.88,122.09,120.20,118.93,114.15,111.65,80.13,37.05$. All the analytical data are in accordance with the reported literature [42].

\subsection{8. (R)-3-(1-(2,6-Dichlorophenyl)-2-nitroethyl)-1H-indole (10h)}

Indole 8a (24 mg, $0.2 \mathrm{mmol})$ and 2,6-dichloronitronitrostyrene $9 \mathrm{~h}$ ( $44 \mathrm{mg}, 0.2 \mathrm{mmol})$ were reacted according to the GP4 to yield product $\mathbf{1 0 q}$ as brown oil (isolated yield $40 \mathrm{mg}$, $60 \%$ ). Enantiomeric excess (ee) was determined by chiral HPLC (Chiracel OD-H column) $\left(75 \% \mathrm{n}\right.$-hexane $\left./ i-\mathrm{PrOH}, 1.0 \mathrm{~mL} / \mathrm{min} ; t_{\text {minor }}=11.59 \mathrm{~min} ; t_{\text {major }}=13.27 \mathrm{~min} ; \lambda=254 \mathrm{~nm}\right)$; $64.1 \%$ ee; $[\alpha]_{\mathrm{D}}^{20}=+91.76^{\circ}\left(c 0.031, \mathrm{CH}_{3} \mathrm{OH}\right) ;{ }^{1} \mathrm{H}-\mathrm{NMR}\left(500 \mathrm{MHz}, \mathrm{CDCl}_{3}\right): \delta(\mathrm{ppm})=8.14$ (s, 1H, NH), $7.42(\mathrm{dq}, J=8.0,0.9 \mathrm{~Hz}, 1 \mathrm{H}, \mathrm{Ar}-\mathbf{H}), 7.37-7.23(\mathrm{~m}, 3 \mathrm{H}, \mathrm{Ar}-\mathbf{H}), 7.21-7.13(\mathrm{~m}$, $3 \mathrm{H}, \mathrm{Ar}-\mathrm{H}), 7.06$ (ddd, $J=8.0,7.1,1.0 \mathrm{~Hz}, 1 \mathrm{H}, \mathrm{Ar}-\mathrm{H}), 6.21(\mathrm{ddd}, J=8.4,7.4,1.2 \mathrm{~Hz}, 1 \mathrm{H}$, $\mathrm{CH}), 5.43\left(\mathrm{dd}, J=12.8,7.4 \mathrm{~Hz}, 1 \mathrm{H}, \mathrm{CH}_{2(\mathrm{a})}\right), 5.36\left(\mathrm{dd}, J=12.8,8.0 \mathrm{~Hz}, 1 \mathrm{H}, \mathrm{CH}_{2(\mathrm{~b})}\right) ;{ }^{13} \mathrm{C}-\mathrm{NMR}$ $\left(126 \mathrm{MHz}, \mathrm{CDCl}_{3}\right): \delta(\mathrm{ppm})=136.17,134.32,130.47,129.90,129.48,126.46,122.71,122.65$, $120.17,119.06,111.63,111.44,76.44,38.03$. All the analytical data are in accordance with the reported literature [41].

\subsection{9. (S)-5-Bromo-3-(1-(4-fluorophenyl)-2-nitroethyl)-1H-indole (10i)}

5-bromoindole $\mathbf{8 b}$ (39 mg, $0.2 \mathrm{mmol}$ ) and 4-floronitrostyrene $9 \mathbf{a}$ ( $34 \mathrm{mg}, 0.2 \mathrm{mmol}$ ) were reacted according to the GP4 to yield product $10 \mathrm{~g}$ as yellow oil (isolated yield $40 \mathrm{mg}, 55 \%$ ). Enantiomeric excess (ee) was determined by chiral HPLC (Chiracel OD-H column) (70\% $n$-hexane $\left./ \mathrm{i}-\mathrm{PrOH}, 1.0 \mathrm{~mL} / \mathrm{min} ; t_{\text {minor }}=9.58 \mathrm{~min} ; t_{\text {major }}=14.14 \mathrm{~min} ; \lambda=254 \mathrm{~nm}\right) ; 77.2 \%$ ee; $[\alpha]_{\mathrm{D}}^{20}=-20.93^{\circ}\left(c 0.051, \mathrm{CH}_{3} \mathrm{OH}\right) ; \mathrm{IR}(\mathrm{KBr}): 3417,1544,1376,1242,1179,1028,743,549,524$ $\mathrm{cm}^{-1} ;{ }^{1} \mathrm{H}-\mathrm{NMR}\left(500 \mathrm{MHz}, \mathrm{CDCl}_{3}\right): \delta(\mathrm{ppm})=8.28(\mathrm{~s}, 1 \mathrm{H}, \mathrm{NH}), 7.55(\mathrm{~d}, J=1.8 \mathrm{~Hz}, 1 \mathrm{H}, \mathrm{Ar}-\mathrm{H})$, 7.34-7.29 (m, 3H, Ar-H), $7.25(\mathrm{~d}, J=8.6 \mathrm{~Hz}, 1 \mathrm{H}, \mathrm{Ar}-\mathrm{H}), 7.09-7.03(\mathrm{~m}, 3 \mathrm{H}, \mathrm{Ar}-\mathbf{H}), 5.14(\mathrm{t}$, $J=8.0 \mathrm{~Hz}, 1 \mathrm{H}, \mathrm{CH}), 5.04\left(\mathrm{dd}, J=12.6,7.8 \mathrm{~Hz}, 1 \mathrm{H}, \mathrm{CH}_{2(\mathrm{a})}\right), 4.91(\mathrm{dd}, J=12.5,8.2 \mathrm{~Hz}, 1 \mathrm{H}$, $\left.\mathrm{CH}_{2(\mathrm{~b})}\right) ;{ }^{13} \mathrm{C}-\mathrm{NMR}\left(126 \mathrm{MHz}, \mathrm{CDCl}_{3}\right) \delta(\mathrm{ppm})=163.23$ and $161.27\left(\mathrm{C}_{1}-\mathrm{F}, J_{\mathrm{C}-\mathrm{F}}=247.21 \mathrm{~Hz}\right)$, $135.2,134.58$ and $134.55\left(\mathrm{C}_{4}-\mathrm{F}, J_{\mathrm{C}-\mathrm{F}}=3.28 \mathrm{~Hz}\right), 129.44$ and $129.37\left(\mathrm{C}_{3}-\mathrm{F}, J_{\mathrm{C}-\mathrm{F}}=8.19 \mathrm{~Hz}\right)$, $127.8,125.9,122.8,121.5,116.17$ and $116.00\left(C_{2}-F, J_{C-F}=21.55 \mathrm{~Hz}\right), 113.9,113.4,113.1,79.5$, 
40.7; LC/MS (ESI): found $363.02[\mathrm{M}+\mathrm{H}]^{+}, \mathrm{C}_{16} \mathrm{H}_{12} \mathrm{BrFN}_{2} \mathrm{O}_{2}$ requires 362.01; anal. calcd. for $\mathrm{C}_{16} \mathrm{H}_{12} \mathrm{BrFN}_{2} \mathrm{O}_{2}$ : C, 52.91; H, 3.33; N, 7.71; found: $\mathrm{C}, 53.01 ; \mathrm{H}, 3.39 ; \mathrm{N}, 7.65$.

3.7.10. (S)-5-Bromo-3-(1-(3-bromophenyl)-2-nitroethyl)-1H-indole (10j)

5-bromoindole $8 \mathbf{b}$ ( $39 \mathrm{mg}, 0.2 \mathrm{mmol}$ ) and 3-bromonitrostyrene $9 \mathbf{b}(46 \mathrm{mg}, 0.2 \mathrm{mmol}$ ) were reacted according to the GP4 to yield product 10h as yellow oil (isolated yield $39 \mathrm{mg}$, $46 \%$ ). Enantiomeric excess (ee) was determined by chiral HPLC (Chiracel OD-H column) $\left(80 \% n\right.$-hexane $\left./ i-\mathrm{PrOH}, 1.0 \mathrm{~mL} / \mathrm{min} ; t_{\mathrm{minor}}=21.41 \mathrm{~min} ; t_{\mathrm{major}}=34.37 \mathrm{~min} ; \lambda=254 \mathrm{~nm}\right)$; $79.5 \% e e ;[\alpha]_{\mathrm{D}}^{20}=-45.13^{\circ}\left(c 0.053, \mathrm{CH}_{3} \mathrm{OH}\right)$; IR (KBr): $3401,1538,1378,1009,814,745,589$, $535,421 \mathrm{~cm}^{-1} ;{ }^{1} \mathrm{H}-\mathrm{NMR}\left(500 \mathrm{MHz}, \mathrm{CDCl}_{3}\right): \delta(\mathrm{ppm})=8.24(\mathrm{~s}, 1 \mathrm{H}, \mathrm{NH}), 7.52(\mathrm{~d}, J=1.9 \mathrm{~Hz}$, $1 \mathrm{H}, \mathrm{Ar}-\mathbf{H}), 7.42-7.37$ (m, 2H, Ar-H), 7.28-7.22 (m, 2H, Ar-H), 7.21-7.16 (m, 2H, Ar-H), $7.03(\mathrm{dd}, J=2.6,0.9 \mathrm{~Hz}, 1 \mathrm{H}, \mathrm{Ar}-\mathbf{H}), 5.07(\mathrm{t}, J=8.0 \mathrm{~Hz}, 1 \mathrm{H}, \mathrm{CH}), 4.97(\mathrm{dd}, J=12.7,8.0 \mathrm{~Hz}$, $\left.1 \mathrm{H}, \mathrm{CH}_{2(\mathrm{a})}\right), 4.86\left(\mathrm{dd}, J=12.7,8.0 \mathrm{~Hz}, 1 \mathrm{H}, \mathrm{CH}_{2(\mathrm{~b})}\right) ;{ }^{13} \mathrm{C}-\mathrm{NMR}\left(126 \mathrm{MHz}, \mathrm{CDCl}_{3}\right): \delta(\mathrm{ppm})$ $=141.2,135.2,131.1,130.8,130.7,127.8,126.5,126.0,123.2,122.9,121.3,113.5,113.3,113.1$, 79.2, 41.0; $\mathrm{LC} / \mathrm{MS}$ (ESI): found $423.01[\mathrm{M}+\mathrm{H}]^{+}, \mathrm{C}_{16} \mathrm{H}_{12} \mathrm{Br}_{2} \mathrm{~N}_{2} \mathrm{O}_{2}$ requires 421.93; anal. calcd. for $\mathrm{C}_{16} \mathrm{H}_{12} \mathrm{Br}_{2} \mathrm{~N}_{2} \mathrm{O}_{2}$ : C, 45.31; $\mathrm{H}, 2.85 ; \mathrm{N}, 6.61$; found: $\mathrm{C}, 45.23 ; \mathrm{H}, 2.96 ; \mathrm{N}, 6.52$.

\subsubsection{1. (S)-5-Bromo-3-(2-nitro-1-(4-(trifluoromethyl)phenyl)ethyl)-1H-indole (10k)}

5-bromoindole $8 \mathbf{b}(39 \mathrm{mg}, 0.2 \mathrm{mmol})$ and 4-trifluoromethynitrostyrene $9 \mathbf{c}(44 \mathrm{mg}$, $0.2 \mathrm{mmol}$ ) were reacted according to the GP4 to yield product 10i as colorless oil (isolated yield $29 \mathrm{mg}, 35 \%$ ). Enantiomeric excess (ee) was determined by chiral HPLC (Chiracel OD-H column) $\left(75 \% n\right.$-hexane $/ i-\mathrm{PrOH}, 1.0 \mathrm{~mL} / \mathrm{min} ; t_{\mathrm{minor}}=11.80 \mathrm{~min} ; t_{\mathrm{major}}=19.82 \mathrm{~min}$; $\lambda=254 \mathrm{~nm}) ; 78.43 \% e e ;[\alpha]_{\mathrm{D}}^{20}=-29.51^{\circ}\left(c 0.056, \mathrm{CH}_{3} \mathrm{OH}\right) ; \mathrm{IR}(\mathrm{KBr}): 3418,1537,1371$, $1247,1103,715,519 \mathrm{~cm}^{-1} ;{ }^{1} \mathrm{H}-\mathrm{NMR}\left(500 \mathrm{MHz}, \mathrm{CDCl}_{3}\right): \delta(\mathrm{ppm})=8.22(\mathrm{~s}, 1 \mathrm{H}, \mathrm{NH}), 7.60(\mathrm{~d}$, $J=8.1 \mathrm{~Hz}, 2 \mathrm{H}, \mathrm{Ar}-\mathrm{H}), 7.53(\mathrm{~d}, J=1.8 \mathrm{~Hz}, 1 \mathrm{H}, \mathrm{Ar}-\mathrm{H}), 7.44(\mathrm{~d}, J=8.1 \mathrm{~Hz}, 2 \mathrm{H}, \mathrm{Ar}-\mathrm{H}), 7.30$ $(\mathrm{dd}, J=8.7,1.9 \mathrm{~Hz}, 1 \mathrm{H}, \mathrm{Ar}-\mathbf{H}), 7.25(\mathrm{~d}, J=8.7 \mathrm{~Hz}, 1 \mathrm{H}, \mathrm{Ar}-\mathbf{H}), 7.07(\mathrm{~d}, J=2.6 \mathrm{~Hz}, 1 \mathrm{H}, \mathrm{Ar}-\mathbf{H})$, $5.20(\mathrm{t}, J=8.0 \mathrm{~Hz}, 1 \mathrm{H}, \mathrm{CH}), 5.04\left(\mathrm{dd}, J=12.8,7.6 \mathrm{~Hz}, 1 \mathrm{H}, \mathrm{CH}_{2(\mathrm{a})}\right), 4.94(\mathrm{dd}, J=12.8,8.4 \mathrm{~Hz}$, $\left.1 \mathrm{H}, \mathrm{CH}_{2(\mathrm{~b})}\right) ;{ }^{13} \mathrm{C}-\mathrm{NMR}\left(126 \mathrm{MHz}, \mathrm{CDCl}_{3}\right): \delta(\mathrm{ppm})=142.9,135.2,128.2,127.7,126.3,126.23$, 126.20, 126.1, 122.9, 121.4, 113.7, 113.3, 113.2, 79.0, 41.1; LC/MS (ESI): found $423.01[\mathrm{M}+\mathrm{H}]^{+}$, $\mathrm{C}_{17} \mathrm{H}_{12} \mathrm{BrF}_{3} \mathrm{~N}_{2} \mathrm{O}_{2}$ requires 421.93; anal. calcd. for $\mathrm{C}_{17} \mathrm{H}_{12} \mathrm{BrF}_{3} \mathrm{~N}_{2} \mathrm{O}_{2}: \mathrm{C}, 49.42 ; \mathrm{H}, 2.93 ; \mathrm{N}$, 6.78; found: C, $49.61 ; \mathrm{H}, 3.07 ; \mathrm{N}, 6.69$.

\subsubsection{2. (S)-5-Bromo-3-(1-(4-methoxyphenyl)-2-nitroethyl)-1H-indole (101)}

5-bromoindole $8 \mathbf{b}$ ( $39 \mathrm{mg}, 0.2 \mathrm{mmol}$ ) and 4-methoxynitrostyrene $9 \mathrm{~d}(36 \mathrm{mg}, 0.2 \mathrm{mmol})$ were reacted according to the GP4 to yield product $10 \mathbf{j}$ as white solid (isolated yield $29 \mathrm{mg}$, $39 \%$ ), m.p. $145-146^{\circ} \mathrm{C}$; Enantiomeric excess (ee) was determined by chiral HPLC (Chiracel OD-H column) $\left(75 \%\right.$-hexane $/ i-\mathrm{PrOH}, 1.0 \mathrm{~mL} / \mathrm{min} ; t_{\text {minor }}=17.33 \mathrm{~min} ; t_{\text {major }}=20.21 \mathrm{~min}$; $\lambda=254 \mathrm{~nm} ; 62.6 \% e e ;[\alpha]_{\mathrm{D}}^{20}=-29.43^{\circ}\left(c 0.053, \mathrm{CH}_{3} \mathrm{OH}\right) ;{ }^{1} \mathrm{H}-\mathrm{NMR}\left(500 \mathrm{MHz}, \mathrm{CDCl}_{3}\right)$ : $\delta(\mathrm{ppm})=8.13(\mathrm{~s}, 1 \mathrm{H}, \mathrm{NH}), 7.53(\mathrm{~d}, J=1.9 \mathrm{~Hz}, 1 \mathrm{H}, \mathrm{Ar}-\mathrm{H}), 7.26(\mathrm{~d}, J=3.4 \mathrm{~Hz}, 1 \mathrm{H}, \mathrm{Ar}-\mathrm{H})$, 7.23-7.18 (m, 3H, Ar-H), 7.06 (dd, J = 2.6, $0.9 \mathrm{~Hz}, 1 \mathrm{H}, \mathrm{Ar}-\mathbf{H}), 6.89-6.83(\mathrm{~m}, 2 \mathrm{H}, \mathrm{Ar}-\mathbf{H})$, $5.07(\mathrm{t}, J=8.0 \mathrm{~Hz}, 1 \mathrm{H}, \mathrm{CH}), 4.99\left(\mathrm{dd}, J=12.3,8.0 \mathrm{~Hz}, 1 \mathrm{H}, \mathrm{CH}_{2(\mathrm{a})}\right), 4.87(\mathrm{dd}, J=12.3,8.0 \mathrm{~Hz}$, $\left.1 \mathrm{H}, \mathrm{CH}_{2(\mathrm{~b})}\right), 3.78\left(\mathrm{~s}, 3 \mathrm{H}, \mathrm{CH}_{3}\right) ;{ }^{13} \mathrm{C}-\mathrm{NMR}\left(126 \mathrm{MHz}, \mathrm{CDCl}_{3}\right): \delta(\mathrm{ppm})=159.2,135.3,130.8$, $128.9,128.0,125.8,122.7,121.7,114.6,114.5,113.4,112.9,79.7,55.4,40.7$. All the analytical data are in accordance with the reported literature [81].

\subsubsection{3. (R)-5-Bromo-3-(2-nitro-1-(2-nitrophenyl)ethyl)-1H-indole (10m)}

5-bromoindole $8 \mathbf{b}$ ( $39 \mathrm{mg}, 0.2 \mathrm{mmol}$ ) and 2-nitronitrostyrene $9 \mathbf{e}$ (39 mg, $0.2 \mathrm{mmol}$ ) were reacted according to the GP4 to yield product 10k as yellow oil (isolated yield $33 \mathrm{mg}$, $42 \%$ ). Enantiomeric excess (ee) was determined by chiral HPLC (Chiracel OD-H column) $\left(75 \% n\right.$-hexane $/ i$-PrOH, $\left.1.0 \mathrm{~mL} / \mathrm{min} ; t_{\text {minor }}=25.65 \mathrm{~min} ; t_{\text {major }}=28.75 \mathrm{~min} ; \lambda=254 \mathrm{~nm}\right)$; $77.69 \% e e ;[\alpha]_{\mathrm{D}}^{20}=+21.38^{\circ}\left(c 0.07, \mathrm{CH}_{3} \mathrm{OH}\right) ; \mathrm{IR}(\mathrm{KBr}): 3419,1548,1513,1339,723,431$ $\mathrm{cm}^{-1} ;{ }^{1} \mathrm{H}-\mathrm{NMR}\left(500 \mathrm{MHz}, \mathrm{CDCl}_{3}\right): \delta(\mathrm{ppm})=8.32(\mathrm{~s}, 1 \mathrm{H}, \mathrm{NH}), 7.92(\mathrm{dd}, J=8.1,1.4 \mathrm{~Hz}, 1 \mathrm{H}$, Ar-H), 7.55-7.49 (m, 1H, Ar-H), 7.46-7.38 (m, 3H, Ar-H), 7.27-7.23 (m, 1H, Ar-H), 7.20 (d, $J=8.6 \mathrm{~Hz}, 1 \mathrm{H}, \mathrm{Ar}-\mathbf{H}), 7.13(\mathrm{~d}, J=2.6 \mathrm{~Hz}, 1 \mathrm{H}, \mathrm{Ar}-\mathbf{H}), 5.83(\mathrm{t}, J=7.7 \mathrm{~Hz}, 1 \mathrm{H}, \mathrm{CH}), 5.10(\mathrm{dd}$, 
$\left.J=13.3,7.0 \mathrm{~Hz}, 1 \mathrm{H}, \mathrm{CH}_{2(\mathrm{a})}\right), 5.03\left(\mathrm{dd}, J=13.3,8.4 \mathrm{~Hz}, 1 \mathrm{H}, \mathrm{CH}_{2(\mathrm{~b})}\right) ;{ }^{13} \mathrm{C}-\mathrm{NMR}(126 \mathrm{MHz}$, $\left.\mathrm{CDCl}_{3}\right): \delta(\mathrm{ppm})=149.6,135.2,133.5,133.4,129.9,129.0,127.7,126.1,125.5,123.5,121.3$, 113.6, 113.1, 112.3, 78.1, 36.4; LC/MS (ESI): found $390.02[\mathrm{M}+\mathrm{H}]^{+}, \mathrm{C}_{16} \mathrm{H}_{12} \mathrm{BrN}_{3} \mathrm{O}_{4}$ requires 389.00; anal. calcd. for $\mathrm{C}_{16} \mathrm{H}_{12} \mathrm{BrN}_{3} \mathrm{O}_{4}: \mathrm{C}, 49.25 ; \mathrm{H}, 3.10 ; \mathrm{N}, 10.77$; found: $\mathrm{C}, 49.33 ; \mathrm{H}, 3.17$; $\mathrm{N}, 10.84$.

\subsubsection{4. (R)-5-Bromo-3-(1-(2,4-dichlorophenyl)-2-nitroethyl)-1H-indole (10n)}

5-bromoindole $8 \mathbf{b}(39 \mathrm{mg}, 0.2 \mathrm{mmol}$ ) and 2,4-dichloronitronitrostyrene $9 \mathrm{f}$ ( $44 \mathrm{mg}$, $0.2 \mathrm{mmol}$ ) were reacted according to the GP4 to yield product 101 as brown oil (isolated yield $31 \mathrm{mg}, 37 \%$ ). Enantiomeric excess (ee) was determined by chiral HPLC (Chiracel OD-H column) $\left(75 \% n\right.$-hexane $/ i$-PrOH, $1.0 \mathrm{~mL} / \mathrm{min} ; t_{\text {minor }}=9.90 \mathrm{~min} ; t_{\text {major }}=20.31 \mathrm{~min}$; $\lambda=254 \mathrm{~nm}) ; 74.6 \% e e ;[\alpha]_{\mathrm{D}}^{20}=-22.88^{\circ}\left(c 0.056, \mathrm{CH}_{3} \mathrm{OH}\right) ; \mathrm{IR}(\mathrm{KBr}): 3417,1542,1456,1348$, $1098,809,742,587 \mathrm{~cm}^{-1}{ }^{1} \mathrm{H}-\mathrm{NMR}\left(500 \mathrm{MHz}, \mathrm{CDCl}_{3}\right): \delta(\mathrm{ppm})=8.26(\mathrm{~s}, 1 \mathrm{H}, \mathrm{NH}), 7.48(\mathrm{dd}$, $J=18.0,2.0 \mathrm{~Hz}, 2 \mathrm{H}, \mathrm{Ar}-\mathbf{H}), 7.28-7.24(\mathrm{~m}, 1 \mathrm{H}, \mathrm{Ar}-\mathbf{H}), 7.21(\mathrm{dd}, J=8.6,1.0 \mathrm{~Hz}, 1 \mathrm{H}, \mathrm{Ar}-\mathbf{H})$, $7.14(\mathrm{dd}, J=8.4,2.1 \mathrm{~Hz}, 1 \mathrm{H}, \mathrm{Ar}-\mathbf{H}), 7.10(\mathrm{dd}, J=2.6,1.1 \mathrm{~Hz}, 1 \mathrm{H}, \mathrm{Ar}-\mathbf{H}), 7.07(\mathrm{dd}, J=8.4$, $1.1 \mathrm{~Hz}, 1 \mathrm{H}, \mathrm{Ar}-\mathbf{H}), 5.59(\mathrm{t}, J=7.9 \mathrm{~Hz}, 1 \mathrm{H}, \mathrm{CH}), 4.92\left(\mathrm{~d}, J=1.9 \mathrm{~Hz}, 1 \mathrm{H}, \mathrm{CH}_{2(\mathrm{a})}\right), 4.91(\mathrm{~d}$, $\left.J=1.1 \mathrm{~Hz}, 1 \mathrm{H}, \mathrm{CH}_{2(\mathrm{~b})}\right) ;{ }^{13} \mathrm{C}-\mathrm{NMR}\left(126 \mathrm{MHz}, \mathrm{CDCl}_{3}\right): \delta(\mathrm{ppm})=135.2,134.8,134.6,134.4$, $130.2,129.8,127.8,126.1,123.3,121.4,113.6,113.1,112.5,77.4,37.5 ; \mathrm{LC} / \mathrm{MS}$ (ESI): found $413.01[\mathrm{M}+\mathrm{H}]^{+}, \mathrm{C}_{16} \mathrm{H}_{11} \mathrm{BrCl}_{2} \mathrm{~N}_{2} \mathrm{O}_{2}$ requires 411.94; anal. calcd. for $\mathrm{C}_{16} \mathrm{H}_{11} \mathrm{BrCl}_{2} \mathrm{~N}_{2} \mathrm{O}_{2}: \mathrm{C}$, $46.41 ; \mathrm{H}, 2.68 ; \mathrm{N}, 6.77$; found: $\mathrm{C}, 46.27 ; \mathrm{H}, 2.57 ; \mathrm{N}, 6.79$.

\subsubsection{5. (S)-5-Bromo-3-(2-nitro-1-(thiophen-2-yl)ethyl)-1H-indole (10o)}

5-bromoindole $8 \mathbf{b}$ (39 mg, $0.2 \mathrm{mmol}$ ) and (E)-2-(2-nitrovinyl)thiophene $9 \mathrm{~g}$ (31 mg, $0.2 \mathrm{mmol}$ ) were reacted according to the GP4 to yield product $10 \mathrm{~m}$ as brown oil (isolated yield $33 \mathrm{mg}, 47 \%$ ). Enantiomeric excess (ee) was determined by chiral HPLC (Chiracel OD-H column) $\left(75 \% n\right.$-hexane $/ i-\mathrm{PrOH}, 1.0 \mathrm{~mL} / \mathrm{min} ; t_{\text {minor }}=12.18 \mathrm{~min} ; t_{\text {major }}=20.57 \mathrm{~min}$; $\lambda=254 \mathrm{~nm}) ; 72.0 \% e e ;[\alpha]_{\mathrm{D}}^{20}=-6.87^{\circ}\left(c 0.081, \mathrm{CH}_{3} \mathrm{OH}\right) ;{ }^{1} \mathrm{H}-\mathrm{NMR}\left(500 \mathrm{MHz}, \mathrm{CDCl}_{3}\right)$ : $\delta(\mathrm{ppm})=8.50(\mathrm{~s}, 1 \mathrm{H}, \mathrm{NH}), 7.62(\mathrm{~d}, J=2.0 \mathrm{~Hz}, 1 \mathrm{H}, \mathrm{Ar}-\mathrm{H}), 7.27(\mathrm{dd}, J=8.7,1.9 \mathrm{~Hz}, 1 \mathrm{H}, \mathrm{Ar}-$ H), 7.22-7.18 (m, 2H, Ar-H), $7.10(\mathrm{~d}, J=2.6 \mathrm{~Hz}, 1 \mathrm{H}, \mathrm{Ar}-\mathbf{H}), 6.97-6.92(\mathrm{~m}, 2 \mathrm{H}, \mathrm{Ar}-\mathbf{H}), 5.38(\mathrm{t}$, $J=7.9 \mathrm{~Hz}, 1 \mathrm{H}, \mathrm{CH}), 5.03-4.93\left(\mathrm{~m}, 2 \mathrm{H}, \mathrm{CH}_{2}\right) ;{ }^{13} \mathrm{C}-\mathrm{NMR}\left(126 \mathrm{MHz}, \mathrm{CDCl}_{3}\right): \delta(\mathrm{ppm})=142.57$, 135.16, 127.56, 127.18, 125.72, 125.46, 125.20, 123.31, 121.41, 113.62, 113.39, 113.16, 79.95, 36.80. All the analytical data are in accordance with the reported literature [42].

\subsubsection{6. (R)-5-Bromo-3-(1-(2,6-dichlorophenyl)-2-nitroethyl)-1H-indole (10p)}

5-Bromoindole $8 \mathbf{b}(39 \mathrm{mg}, 0.2 \mathrm{mmol})$ and 2,6-dichloronitronitrostyrene $9 \mathrm{~h}(44 \mathrm{mg}$, $0.2 \mathrm{mmol}$ ) were reacted according to the GP4 to yield product $10 \mathrm{p}$ as brown oil (isolated yield $43 \mathrm{mg}, 52 \%$ ). Enantiomeric excess (ee) was determined by chiral HPLC (Chiracel OD-H column) $\left(75 \% n\right.$-hexane $/ i-\mathrm{PrOH}, 1.0 \mathrm{~mL} / \mathrm{min} ; t_{\text {minor }}=10.29 \mathrm{~min} ; t_{\text {major }}=11.29 \mathrm{~min}$; $\lambda=254 \mathrm{~nm}) ; 60.1 \% e e ;[\alpha]_{\mathrm{D}}^{20}=+32.64^{\circ}\left(c 0.034, \mathrm{CH}_{3} \mathrm{OH}\right) ; \mathrm{IR}(\mathrm{KBr}): 3415,1549,1463,1356$, $1109,822,734,605,541,424 \mathrm{~cm}^{-1},{ }^{1} \mathrm{H}-\mathrm{NMR}\left(500 \mathrm{MHz}, \mathrm{CDCl}_{3}\right): \delta(\mathrm{ppm})=8.85(\mathrm{~s}, 1 \mathrm{H}, \mathrm{NH})$, $7.50(\mathrm{~s}, 1 \mathrm{H}, \mathrm{Ar}-\mathbf{H}), 7.32(\mathrm{~s}, 1 \mathrm{H}, \mathrm{Ar}-\mathbf{H}), 7.26(\mathrm{~s}, 1 \mathrm{H}, \mathrm{Ar}-\mathbf{H}), 7.22-7.19(\mathrm{~m}, 2 \mathrm{H}, \mathrm{Ar}-\mathbf{H}), 7.18-7.13$ $(\mathrm{m}, 2 \mathrm{H}, \mathrm{Ar}-\mathrm{H}), 6.12(\mathrm{td}, J=7.7,1.2 \mathrm{~Hz}, 1 \mathrm{H}, \mathrm{CH}), 5.39\left(\mathrm{dd}, J=12.9,7.7 \mathrm{~Hz}, 1 \mathrm{H}, \mathrm{CH}_{2(\mathrm{a})}\right)$, $5.29\left(\mathrm{dd}, J=12.9,7.8 \mathrm{~Hz}, 1 \mathrm{H}, \mathrm{CH}_{2(\mathrm{~b})}\right) ;{ }^{13} \mathrm{C}-\mathrm{NMR}\left(126 \mathrm{MHz}, \mathrm{CDCl}_{3}\right): \delta(\mathrm{ppm})=134.86$, 133.93, 130.06129.64, 128.19, 125.38, 124.10, 121.60, 114.17, 113.25, 112.94, 111.02, 76.27, 37.77; $\mathrm{LC} / \mathrm{MS}$ (ESI): found $412.98[\mathrm{M}+\mathrm{H}]^{+}, \mathrm{C}_{16} \mathrm{H}_{11} \mathrm{BrCl}_{2} \mathrm{~N}_{2} \mathrm{O}_{2}$ requires 411.94; Anal. calcd. for $\mathrm{C}_{16} \mathrm{H}_{11} \mathrm{BrCl}_{2} \mathrm{~N}_{2} \mathrm{O}_{2}$ : C, 46.41; H, 2.68; N, 6.77; Found: $\mathrm{C}, 46.36 ; \mathrm{H}, 2.74 ; \mathrm{N}, 6.63$.

\subsubsection{7. (S)-5-Fluoro-3-(2-nitro-1-(thiophen-2-yl)ethyl)-1H-indole(10q)}

5-Fluoroindole $8 \mathrm{c}(27 \mathrm{mg}, 0.2 \mathrm{mmol})$ and (E)-2-(2-nitrovinyl)thiophene $9 \mathrm{~g}(31 \mathrm{mg}$, $0.2 \mathrm{mmol}$ ) were reacted according to the GP4 to yield product $10 \mathrm{o}$ as brown oil (isolated yield $33 \mathrm{mg}, 57 \%$ ). Enantiomeric excess (ee) was determined by chiral HPLC (Chiracel OD-H column) $\left(75 \% n\right.$-hexane $/ i-\mathrm{PrOH}, 1.0 \mathrm{~mL} / \mathrm{min} ; t_{\text {minor }}=11.81 \mathrm{~min} ; t_{\text {major }}=13.42 \mathrm{~min}$; $\lambda=254 \mathrm{~nm}) ; 66.0 \% e e ;[\alpha]_{\mathrm{D}}^{20}=+36.97^{\circ}\left(c 0.035, \mathrm{CH}_{3} \mathrm{OH}\right) ; \mathrm{IR}(\mathrm{KBr}): 3417,1547,1469,1343$, $1205,827,731,541 \mathrm{~cm}^{-1} ;{ }^{1} \mathrm{H}-\mathrm{NMR}\left(500 \mathrm{MHz}, \mathrm{CDCl}_{3}\right): \delta(\mathrm{ppm})=8.18(\mathrm{~s}, 1 \mathrm{H}, \mathrm{NH}), 7.40(\mathrm{dd}$, 
$J=8.7,5.2 \mathrm{~Hz}, 1 \mathrm{H}, \mathrm{Ar}-\mathbf{H}), 7.21(\mathrm{dd}, J=5.1,1.3 \mathrm{~Hz}, 1 \mathrm{H}, \mathrm{Ar}-\mathbf{H}), 7.13-7.08(\mathrm{~m}, 1 \mathrm{H}, \mathrm{Ar}-\mathbf{H})$, $7.04(\mathrm{dd}, J=9.4,2.3 \mathrm{~Hz}, 1 \mathrm{H}, \mathrm{Ar}-\mathrm{H}), 7.00-6.92(\mathrm{~m}, 2 \mathrm{H}, \mathrm{Ar}-\mathrm{H}), 6.87(\mathrm{td}, J=9.2,2.3 \mathrm{~Hz}$, $1 \mathrm{H}, \mathrm{Ar}-\mathrm{H}), 5.43(\mathrm{t}, J=7.9 \mathrm{~Hz}, 1 \mathrm{H}, \mathrm{CH}), 5.05-4.96\left(\mathrm{~m}, 2 \mathrm{H}, \mathrm{CH}_{2}\right) ;{ }^{13} \mathrm{C}-\mathrm{NMR}(126 \mathrm{MHz}$, $\left.\mathrm{CDCl}_{3}\right): \delta(\mathrm{ppm})=161.31$ and $159.41\left(\mathrm{C}_{1}-\mathrm{F}, J_{\mathrm{C}-\mathrm{F}}=239.40 \mathrm{~Hz}\right), 142.84,136.59,136.49,127.15$, $125.46,125.18,122.48,122.28$ and $122.25\left(\mathrm{C}_{4}-\mathrm{F}, J_{\mathrm{C}-\mathrm{F}}=3.53 \mathrm{~Hz}\right), 119.84$ and $119.76\left(\mathrm{C}_{3}-\mathrm{F}\right.$, $\left.J_{\text {C-F }}=10.04 \mathrm{~Hz}\right), 114.35,109.22$ and $109.02\left(\mathrm{C}_{2}-\mathrm{F}, J_{\mathrm{C}-\mathrm{F}}=23.94 \mathrm{~Hz}\right), 80.09,36.99 ; \mathrm{LC} / \mathrm{MS}$ (ESI): found $291.10[\mathrm{M}+\mathrm{H}]^{+}, \mathrm{C}_{14} \mathrm{H}_{11} \mathrm{FN}_{2} \mathrm{O}_{2} \mathrm{~S}$ requires 290.05; Anal. calcd. for $\mathrm{C}_{14} \mathrm{H}_{11} \mathrm{FN}_{2} \mathrm{O}_{2} \mathrm{~S}: \mathrm{C}$, 57.92; H, 3.82; N, 9.65; Found: C, 58.11; H, 3.93; N, 9.52.

\subsubsection{8. (R)-3-(1-(2,6-Dichlorophenyl)-2-nitroethyl)-1H-indole (10r)}

5-Fluoroindole $8 \mathrm{c}(27 \mathrm{mg}, 0.2 \mathrm{mmol})$ and 2,6-dichloronitronitrostyrene $9 \mathrm{~h}(44 \mathrm{mg}$, $0.2 \mathrm{mmol}$ ) were reacted according to the GP4 to yield product 10r as brown oil (isolated yield $32 \mathrm{mg}, 45 \%$ ). Enantiomeric excess (ee) was determined by chiral HPLC (Chiracel OD-H column) $\left(75 \% n\right.$-hexane $/ i-\mathrm{PrOH}, 1.0 \mathrm{~mL} / \mathrm{min} ; t_{\text {minor }}=7.99 \mathrm{~min} ; t_{\text {major }}=10.29 \mathrm{~min}$; $\lambda=254 \mathrm{~nm}) ; 24.3 \% e e ;[\alpha]_{\mathrm{D}}^{20}=+65.97\left(c 0.029, \mathrm{CH}_{3} \mathrm{OH}\right) ; \mathrm{IR}(\mathrm{KBr}): 3418,1551,1472,1371$, $1101,819,735 \mathrm{~cm}^{-1},{ }^{1} \mathrm{H}-\mathrm{NMR}\left(500 \mathrm{MHz}, \mathrm{CDCl}_{3}\right): \delta(\mathrm{ppm})=8.20(\mathrm{~s}, 1 \mathrm{H}, \mathrm{NH}), 7.43-7.22(\mathrm{~m}$, $3 \mathrm{H}, \mathrm{Ar}-\mathbf{H}), 7.18-7.12(\mathrm{~m}, 2 \mathrm{H}, \mathrm{Ar}-\mathbf{H}), 7.02(\mathrm{dd}, J=9.4,2.3 \mathrm{~Hz}, 1 \mathrm{H}, \mathrm{Ar}-\mathbf{H}), 6.81$ (ddd, J = 9.5, $8.8,2.3 \mathrm{~Hz}, 1 \mathrm{H}, \mathrm{Ar}-\mathbf{H}), 6.17(\mathrm{td}, J=7.6,1.2 \mathrm{~Hz}, 1 \mathrm{H}, \mathrm{CH}), 5.42\left(\mathrm{dd}, J=12.8,7.6 \mathrm{~Hz}, 1 \mathrm{H}, \mathrm{CH}_{2(\mathrm{a})}\right)$, $5.31\left(\mathrm{dd}, J=12.9,7.7 \mathrm{~Hz}, 1 \mathrm{H}, \mathrm{CH}_{2(\mathrm{~b})}\right) ;{ }^{13} \mathrm{C}-\mathrm{NMR}\left(126 \mathrm{MHz}, \mathrm{CDCl}_{3}\right): \delta(\mathrm{ppm})=161.13$ and $159.23\left(\mathrm{C}_{1}-\mathrm{F}, J_{\mathrm{C}-\mathrm{F}}=239.14 \mathrm{~Hz}\right), 136.22$ and $136.12\left(\mathrm{C}_{5}-\mathrm{F}, J_{\mathrm{C}-\mathrm{F}}=10.34 \mathrm{~Hz}\right), 134.11,129.61$, $123.04,122.94$ and $122.91\left(\mathrm{C}_{4}-\mathrm{F}, J_{\mathrm{C}-\mathrm{F}}=3.65 \mathrm{~Hz}\right), 119.87$ and $119.79\left(\mathrm{C}_{3}-\mathrm{F}, J_{\mathrm{C}-\mathrm{F}}=10.21 \mathrm{~Hz}\right)$, 111.85, 109.05 and $108.86\left(\mathrm{C}_{6}-\mathrm{F}, J_{\mathrm{C}-\mathrm{F}}=24.57 \mathrm{~Hz}\right), 97.85$ and $97.64\left(\mathrm{C}_{2}-\mathrm{F}, J_{\mathrm{C}-\mathrm{F}}=25.96 \mathrm{~Hz}\right)$, 76.39, 37.92; LC/MS (ESI): found $353.10[\mathrm{M}+\mathrm{H}]^{+}, \mathrm{C}_{16} \mathrm{H}_{11} \mathrm{Cl}_{2} \mathrm{FN}_{2} \mathrm{O}_{2}$ requires 352.01; anal. calcd. for $\mathrm{C}_{16} \mathrm{H}_{11} \mathrm{Cl}_{2} \mathrm{FN}_{2} \mathrm{O}_{2}$ : C, 54.41; H, 3.14; N, 7.93; found: $\mathrm{C}, 54.58 ; \mathrm{H}, 3.08 ; \mathrm{N}, 8.03$.

\subsubsection{9. (S)-1-Ethyl-3-(1-(4-fluorophenyl)-2-nitroethyl)-1H-indole (10s)}

1-Ethyl-1H-indole $8 \mathbf{d}(29 \mathrm{mg}, 0.2 \mathrm{mmol})$ and 4-floronitrostyrene $9 \mathrm{a}(34 \mathrm{mg}, 0.2 \mathrm{mmol})$ were reacted according to the GP4 to yield product 10s as yellow oil (isolated yield $46 \mathrm{mg}$, $73 \%$ ). Enantiomeric excess (ee) was determined by chiral HPLC (Chiracel OD-H column) $\left(70 \% n\right.$-hexane $\left./ i-\mathrm{PrOH}, 1.0 \mathrm{~mL} / \mathrm{min} ; t_{\text {minor }}=16.38 \mathrm{~min} ; t_{\text {major }}=34.92 \mathrm{~min} ; \lambda=254 \mathrm{~nm}\right) ;$ $35.2 \% e e ;[\alpha]_{\mathrm{D}}^{20}=+44.27^{\circ}\left(c 0.022, \mathrm{CH}_{3} \mathrm{OH}\right)$; IR (KBr): 3418, 1557, 1349, 1174, 739, $573 \mathrm{~cm}^{-1}$; ${ }^{1} \mathrm{H}-\mathrm{NMR}\left(500 \mathrm{MHz}, \mathrm{CDCl}_{3}\right): \delta(\mathrm{ppm})=7.42(\mathrm{~d}, J=7.9 \mathrm{~Hz}, 1 \mathrm{H}, \mathrm{Ar}-\mathbf{H}), 7.36-7.29(\mathrm{~m}, 3 \mathrm{H}$, Ar-H), 7.25-7.21 (m, 1H, Ar-H), 7.08 (ddd, J = 8.0, 7.0, 1.0 Hz, 1H, Ar-H), $7.02(\mathrm{t}, J=8.6 \mathrm{~Hz}$, 2H, Ar-H), $6.92(\mathrm{~d}, J=0.9 \mathrm{~Hz}, 1 \mathrm{H}, \mathrm{Ar}-\mathbf{H}), 5.18(\mathrm{dd}, J=8.7,7.4 \mathrm{~Hz}, 1 \mathrm{H}, \mathrm{CH}), 5.06(\mathrm{dd}$, $\left.J=12.5,7.2 \mathrm{~Hz}, 1 \mathrm{H}, \mathrm{CH}_{2(\mathrm{a})}\right), 4.91\left(\mathrm{dd}, J=12.5,8.9 \mathrm{~Hz}, 1 \mathrm{H}, \mathrm{CH}_{2(\mathrm{~b})}\right), 4.14(\mathrm{q}, J=7.3 \mathrm{~Hz}, 2 \mathrm{H}$, $\left.\mathrm{CH}_{2}\right), 1.45\left(\mathrm{t}, J=7.3 \mathrm{~Hz}, 3 \mathrm{H}, \mathrm{CH}_{3}\right) ;{ }^{13} \mathrm{C}-\mathrm{NMR}\left(126 \mathrm{MHz}, \mathrm{CDCl}_{3}\right): \delta(\mathrm{ppm})=163.17$ and 161.21 $\left(\mathrm{C}_{1}-\mathrm{F}, J_{\mathrm{C}-\mathrm{F}}=246.71 \mathrm{~Hz}\right), 136.50,135.28$ and $135.26\left(\mathrm{C}_{4}-\mathrm{F}, J_{\mathrm{C}-\mathrm{F}}=3.15 \mathrm{~Hz}\right), 129.52,129.46\left(\mathrm{C}_{3}-\mathrm{F}\right.$, $\left.J_{\mathrm{C}-\mathrm{F}}=8.06 \mathrm{~Hz}\right), 126.66,124.56,122.34,119.62,119.14,116.02,115.85\left(\mathrm{C}_{2}-\mathrm{F}, J_{\mathrm{C}-\mathrm{F}}=21.55 \mathrm{~Hz}\right)$, 112.81, 109.79, 79.73, 41.19, 41.06, 15.55; LC/MS (ESI): found $313.10[\mathrm{M}+\mathrm{H}]^{+}, \mathrm{C}_{18} \mathrm{H}_{17} \mathrm{FN}_{2} \mathrm{O}_{2}$ requires 312.13; anal. calcd. for $\mathrm{C}_{18} \mathrm{H}_{17} \mathrm{FN}_{2} \mathrm{O}_{2}$ : $\mathrm{C}, 69.22 ; \mathrm{H}, 5.49 ; \mathrm{N}, 8.97$; found: $\mathrm{C}, 69.34 ; \mathrm{H}$, $5.43 ; \mathrm{N}, 8.85$.

\subsubsection{0. (S)-1-Ethyl-3-(1-(4-methoxyphenyl)-2-nitroethyl)-1H-indole (10t)}

1-Ethyl-1H-indole $8 \mathrm{~d}$ (29 mg, $0.2 \mathrm{mmol}$ ) and 4-methoxynitrostyrene $9 \mathrm{~d}$ (36 mg, $0.2 \mathrm{mmol}$ ) were reacted according to the GP4 to yield product 10t as yellow oil (isolated yield $49 \mathrm{mg}$, $76 \%$ ). Enantiomeric excess (ee) was determined by chiral HPLC (Chiracel OD-H column) $\left(70 \% n\right.$-hexane $\left./ i-\mathrm{PrOH}, 1.0 \mathrm{~mL} / \mathrm{min} ; t_{\text {minor }}=20.94 \mathrm{~min} ; t_{\text {major }}=35.44 \mathrm{~min} ; \lambda=254 \mathrm{~nm}\right)$; $26.74 \% e e ;[\alpha]_{\mathrm{D}}^{20}=+20.60^{\circ}\left(c 0.024, \mathrm{CH}_{3} \mathrm{OH}\right) ; \mathrm{IR}(\mathrm{KBr}): 3417,152,1337,1171,741,534 \mathrm{~cm}^{-1}$; ${ }^{1} \mathrm{H}-\mathrm{NMR}\left(500 \mathrm{MHz}, \mathrm{CDCl}_{3}\right): \delta(\mathrm{ppm})=7.44(\mathrm{~d}, J=8.0 \mathrm{~Hz}, 1 \mathrm{H}, \mathrm{Ar}-\mathrm{H}), 7.32(\mathrm{~d}, J=8.3 \mathrm{~Hz}, 1 \mathrm{H}$, Ar-H), 7.27-7.24 (m, 2H, Ar-H), 7.21 (t, J = 7.0 Hz, 1H, Ar-H), 7.06 (t, J = 7.5 Hz, 1H, Ar-H), $6.90(\mathrm{~s}, 1 \mathrm{H}, \mathrm{Ar}-\mathbf{H}), 6.85(\mathrm{~d}, J=8.7 \mathrm{~Hz}, 2 \mathrm{H}, \mathrm{Ar}-\mathbf{H}), 5.13(\mathrm{t}, J=8.0 \mathrm{~Hz}, 1 \mathrm{H}, \mathrm{CH}), 5.03(\mathrm{dd}$, $\left.J=12.3,7.3 \mathrm{~Hz}, 1 \mathrm{H}, \mathrm{CH}_{2(\mathrm{a})}\right), 4.89\left(\mathrm{dd}, J=12.4,8.8 \mathrm{~Hz}, 1 \mathrm{H}, \mathrm{CH}_{2(\mathrm{~b})}\right), 4.12(\mathrm{q}, J=7.3 \mathrm{~Hz}, 2 \mathrm{H}$, $\left.\mathrm{CH}_{2}\right), 3.77\left(\mathrm{~s}, 3 \mathrm{H}, \mathrm{CH}_{3}\right), 1.43\left(\mathrm{t}, J=7.3 \mathrm{~Hz}, 3 \mathrm{H}, \mathrm{CH}_{3}\right) ;{ }^{13} \mathrm{C}-\mathrm{NMR}\left(126 \mathrm{MHz}, \mathrm{CDCl}_{3}\right): \delta(\mathrm{ppm})$ $=158.99,136.49,131.51,128.94,126.82,124.62,122.18,119.48,119.28,114.38,113.33,109.70$, 
55.37, 41.14, 41.06, 15.55; $\mathrm{LC} / \mathrm{MS}$ (ESI): found $325.20[\mathrm{M}+\mathrm{H}]^{+}, \mathrm{C}_{19} \mathrm{H}_{20} \mathrm{~N}_{2} \mathrm{O}_{3}$ requires 324.15; anal. calcd. for $\mathrm{C}_{19} \mathrm{H}_{20} \mathrm{~N}_{2} \mathrm{O}_{3}$ : C, 70.35; H, 6.21; N, 8.64; found: $\mathrm{C}, 70.19 ; \mathrm{H}, 6.13 ; \mathrm{N}, 8.54$.

\section{Large-Scale Synthesis of (S)-3-(1-(4-Fluorophenyl)-2-nitroethyl)-1H-indole (10a)}

An oven-dried 50-mL round bottom flask equipped with a condenser under nitrogen atmosphere was charged with ligand $\mathbf{L} 5(100 \mathrm{mg}, 0.3 \mathrm{mmol}, 15 \% \mathrm{~mol}), \mathrm{Cu}(\mathrm{OTf})_{2}(110 \mathrm{mg}$, $0.3 \mathrm{mmol}, 15 \mathrm{~mol} \%)$ and dry toluene $(20 \mathrm{~mL})$. The mixture was then stirred at reflux for $2 \mathrm{~h}$. After cooling to room temperature, 4-floronitrostyrene (9a) $(334 \mathrm{mg}, 2.0 \mathrm{mmol})$ and $4 \mathrm{~A}^{\circ}$ molecular sieves were added. Then, the mixture was stirred for another $30 \mathrm{~min}$, followed by the addition of indole $8 \mathrm{a}(234 \mathrm{mg}, 2.0 \mathrm{mmol})$. The reaction was then left stirring for $48 \mathrm{~h}$ at room temperature. The solvent was removed under reduced pressure, and the crude product was isolated by flash column chromatography on silica gel, eluting with ethylacetate $/ n$-hexane $(2: 8, v / v)$ to afford a pure Friedel-Crafts product (10a) isolated yield of $76 \%(432 \mathrm{mg}$ ) with $77.2 \%$ enantiomeric excess (ee). Enantiomeric excess (ee) was determined by chiral HPLC (Chiracel OD-H column) $(70 \%$-hexane $/ i-\mathrm{PrOH}, 1.0 \mathrm{~mL} / \mathrm{min}$; $\left.t_{\text {major }}=25.09 \mathrm{~min} ; t_{\text {minor }}=30.30 \mathrm{~min} ; \lambda=254 \mathrm{~nm}\right) ; 77.2 \% e e ;{ }^{1} \mathrm{H}-\mathrm{NMR}\left(500 \mathrm{MHz}, \mathrm{CDCl}_{3}\right)$ : $\delta(\mathrm{ppm})=8.13(\mathrm{~s}, 1 \mathrm{H}, \mathrm{NH}), 7.47-7.40(\mathrm{~m}, 1 \mathrm{H}, \mathrm{Ar}-\mathrm{H}), 7.35(\mathrm{~s}, 1 \mathrm{H}, \mathrm{Ar}-\mathrm{H}), 7.32-7.28(\mathrm{~m}$, $2 \mathrm{H}, \mathrm{Ar}-\mathrm{H}), 7.23(\mathrm{ddd}, J=8.2,7.0,1.2 \mathrm{~Hz}, 1 \mathrm{H}, \mathrm{Ar}-\mathrm{H}), 7.11(\mathrm{ddd}, J=8.1,6.9,1.0 \mathrm{~Hz}, 1 \mathrm{H}$, Ar-H), 7.04-6.96 (m, 3H, Ar-H), $5.19(\mathrm{t}, J=8.0 \mathrm{~Hz}, 1 \mathrm{H}, \mathrm{CH}), 5.05(\mathrm{dd}, J=12.5,7.5 \mathrm{~Hz}, 1 \mathrm{H}$, $\left.\mathrm{CH}_{2(\mathrm{a})}\right), 4.90\left(\mathrm{dd}, J=12.5,8.6 \mathrm{~Hz}, 1 \mathrm{H}, \mathrm{CH}_{2(\mathrm{~b})}\right) ;{ }^{13} \mathrm{C}-\mathrm{NMR}\left(126 \mathrm{MHz}, \mathrm{CDCl}_{3}\right): \delta(\mathrm{ppm})=163.1$ and $161.18\left(\mathrm{C}_{1}-\mathrm{F}, J_{\mathrm{C}-\mathrm{F}}=246.58 \mathrm{~Hz}\right), 136.6,135.07$ and $135.04\left(\mathrm{C}_{4}-\mathrm{F}, J_{\mathrm{C}-\mathrm{F}}=3.15 \mathrm{~Hz}\right), 129.50$ and $129.44\left(\mathrm{C}_{3}-\mathrm{F}, J_{\mathrm{C}-\mathrm{F}}=7.94 \mathrm{~Hz}\right), 126.0,122.9,121.6,120.1,118.9,115.99$ and $115.82\left(\mathrm{C}_{2}-\mathrm{F}\right.$, $\left.J_{\mathrm{C}-\mathrm{F}}=21.67 \mathrm{~Hz}\right), 114.2,111.6,79.6,41.0$.

\section{Conclusions}

In summary, we have synthesized new $C_{2}$-symmetric 2,5-bis(oxazolinyl)thiophene and 2,5-bis(imidazolinyl)thiophene ligands based on thiophene systems and successfully tested them in asymmetric Friedel-Crafts alkylation reactions of indole with trans $\beta$-nitroolefins. Our newly developed catalytic system $\left(15 \mathrm{~mol} \%\right.$ of $\mathbf{L} 5: \mathrm{Cu}(\mathrm{OTf})_{2}$ in toluene at $\left.25^{\circ} \mathrm{C}\right)$ was found to be applicable in inducing chirality into nitroalkylated indoles with low to good yields (35-76\%) and low to good enantioselectivity (21-81\%) at room temperature. On the basis of the screening performed, this methodology could be an alternative tool for asymmetric Friedel-Crafts reactions using this catalytic system. The advantage of this catalytic system is that it is easy to prepare the chiral ligands from the widely accessible thiophene precursor, and the reaction can also be performed at room temperature as compared to other catalytic system carried out at lower temperatures. There is an ongoing research project to explore more utilities for these new chiral thiophene ligands and their applications in asymmetric transformation, and its outcome will be communicated soon in future.

Supplementary Materials: Page S4-S35: ${ }^{1} \mathrm{H}-\mathrm{NMR}$ and ${ }^{13} \mathrm{C}-\mathrm{NMR}$ for compounds 4a-c, L1-L5 and 10a-t and chiral HPLC analysis for compound 10a-t.

Author Contributions: Conceptualization, A.M.A.-M. and A.B.; supervision, A.M.A.-M., A.B. and M.S.I.; methodology, A.S.A., M.S.I. and A.M.A.-M.; validation, M.S.I., A.S.A., S.A. and A.M.A.-M.; formal analysis, A.S.A., M.S.I., S.A. and M.H.; investigation, A.S.A., M.S.I. and S.A.; resources, A.M.A.M. and A.B.; data curation, A.S.A., M.S.I., A.B. and M.H.; writing-original draft preparation, M.S.I., A.B. and A.S.A.; writing—review and editing, M.S.I., A.B., A.M.A.-M. and M.H.; visualization, A.B., M.S.I., S.A. and M.H.; project administration, A.M.A.-M. and A.B.; funding acquisition, A.M.A.-M. and A.B.; software, A.S.A., M.S.I., A.B. and M.H. All authors have read and agreed to the published version of the manuscript.

Funding: Researchers Supporting Project number (RSP-2021/64), King Saud University, Riyadh, Saudi Arabia.

Institutional Review Board Statement: Not applicable. 
Informed Consent Statement: Not applicable.

Data Availability Statement: The data presented in this study are available in Supplementary Materials.

Acknowledgments: The authors would like to extend their sincere appreciation to the Researchers Supporting Project (RSP-2021/64), King Saud University, Riyadh, Saudi Arabia.

Conflicts of Interest: The authors declare no conflict of interest.

\section{References}

1. Cai, F.; Pu, X.; Qi, X.; Lynch, V.; Radha, A.; Ready, J.M. Chiral allene-containing phosphines in asymmetric catalysis. J. Am. Chem. Soc. 2011, 133, 18066-18069. [CrossRef] [PubMed]

2. Leeuwen, P.W.v.; Kamer, P.C.; Claver, C.; Pamies, O.; Dieguez, M. Phosphite-containing ligands for asymmetric catalysis. Chem. Rev. 2011, 111, 2077-2118. [CrossRef] [PubMed]

3. Fernández-Pérez, H.; Etayo, P.; Panossian, A.; Vidal-Ferran, A. Phosphine- phosphinite and phosphine- phosphite ligands: Preparation and applications in asymmetric catalysis. Chem. Rev. 2011, 111, 2119-2176. [CrossRef] [PubMed]

4. Banerjee, D.; Buzas, A.K.; Besnard, C.1.; Kündig, E.P. Chiral n-heterocyclic carbene gold complexes: Synthesis, properties, and application in asymmetric catalysis. Organometallics 2012, 31, 8348-8354. [CrossRef]

5. Yoon, M.; Srirambalaji, R.; Kim, K. Homochiral metal-organic frameworks for asymmetric heterogeneous catalysis. Chem. Rev. 2012, 112, 1196-1231. [CrossRef]

6. Chen, X.; Lu, Z. Recent advances in chiral imino-containing ligands for metal-catalyzed asymmetric transformations. Org. Biomol. Chem. 2017, 15, 2280-2306. [CrossRef]

7. Pellissier, H. Recent developments in enantioselective iron-catalyzed transformations. Coord. Chem. Rev. 2019, 386, 1-31. [CrossRef]

8. Barakat, A.; El-Faham, A.; Haukka, M.; Al-Majid, A.M.; Soliman, S.M. S-triazine pincer ligands: Synthesis of their metal complexes, coordination behavior, and applications. Appl. Organomet. Chem. 2021, 35, e6317. [CrossRef]

9. Kagan, H.B.; Gopalaiah, K. Early history of asymmetric synthesis: Who are the scientists who set up the basic principles and the first experiments? New J. Chem. 2011, 35, 1933-1937. [CrossRef]

10. Oliveira, V.d.G.; Cardoso, M.F.d.C.; Forezi, L.d.S.M. Organocatalysis: A brief overview on its evolution and applications. Catalysts 2018, 8, 605. [CrossRef]

11. Pellissier, H. Asymmetric organocatalysis. Tetrahedron 2007, 38, 9267-9331. [CrossRef]

12. Itoh, T.; Hanefeld, U. Enzyme catalysis in organic synthesis. Green Chem. 2017, 19, 331-332. [CrossRef]

13. Sheldon, R.A.; Brady, D.; Bode, M.L. The hitchhiker's guide to biocatalysis: Recent advances in the use of enzymes in organic synthesis. Chem. Sci. 2020, 11, 2587-2605. [CrossRef]

14. Pàmies, O.; Bäckvall, J.-E. Combination of enzymes and metal catalysts. A powerful approach in asymmetric catalysis. Chem. Rev. 2003, 103, 3247-3262. [CrossRef]

15. Choi, J.; Fu, G.C. Catalytic asymmetric synthesis of secondary nitriles via stereoconvergent negishi arylations and alkenylations of racemic $\alpha$-bromonitriles. J. Am. Chem. Soc. 2012, 134, 9102-9105. [CrossRef]

16. Kalek, M.; Fu, G.C. Phosphine-catalyzed doubly stereoconvergent $\gamma$-additions of racemic heterocycles to racemic allenoates: The catalytic enantioselective synthesis of protected $\alpha, \alpha$-disubstituted $\alpha$-amino acid derivatives. J. Am. Chem. Soc. 2015, 137, 9438-9442. [CrossRef]

17. Park, J.K.; Lackey, H.H.; Ondrusek, B.A.; McQuade, D.T. Stereoconvergent synthesis of chiral allylboronates from an e/z mixture of allylic aryl ethers using a 6-NHC-Cu(I) catalyst. J. Am. Chem. Soc. 2011, 133, 2410-2413. [CrossRef]

18. Li, L.; Chen, Z.; Zhang, X.; Jia, Y. Divergent strategy in natural product total synthesis. Chem. Rev. 2018, 118, 3752-3832. [CrossRef]

19. Shimokawa, J. Divergent strategy in natural product total synthesis. Tetrahedron Lett. 2014, 55, 6156-6162. [CrossRef]

20. Krautwald, S.; Carreira, E.M. Stereodivergence in asymmetric catalysis. J. Am. Chem. Soc. 2017, 139, 5627-5639. [CrossRef]

21. Pellissier, H. Enantioselective vanadium-catalyzed transformations. An update. Coord. Chem. Rev. 2020, 418, 213395. [CrossRef]

22. Roberts, R.M.; Khalaf, A.A. Friedel-Crafts Alkylation Chemistry: A Century of Discovery; Marcel Dekker Incorporated: New York, NY, USA, 1984; Volume 10.

23. Olah, G.A. Friedel-Crafts and related reactions. In Across Conventional Lines: Selected Papers of George a Olah Volume 1; World Scientific: Singapore, 2003; pp. 109-118.

24. Bandini, M.; Melloni, A.; Umani-Ronchi, A. New catalytic approaches in the stereoselective Friedel-Crafts alkylation reaction. Angew. Chem. Int. Ed. 2004, 43, 550-556. [CrossRef]

25. Bandini, M.; Eichholzer, A.; Umani-Ronchi, A. An update on catalytic enantioselective alkylations of indoles. Mini Rev. Org. Chem. 2007, 4, 115-124. [CrossRef]

26. Bi, X.; Zhang, Q.; Gu, Z. Transition-metal-catalyzed carbon-carbon bond activation in asymmetric synthesis. Chin. J. Chem. 2021, 39, 1397-1412. [CrossRef]

27. Barakat, A.; Islam, M.S.; Al Majid, A.M.; Al-Othman, Z.A. Highly enantioselective Friedel-Crafts alkylation of indoles with $\alpha$, $\beta$-unsaturated ketones with simple $\mathrm{Cu}(\mathrm{II})$-oxazoline-imidazoline catalysts. Tetrahedron 2013, 69, 5185-5192. [CrossRef] 
28. Liu, L.; Ma, H.; Xiao, Y.; Du, F.; Qin, Z.; Li, N.; Fu, B. Highly enantioselective Friedel-Crafts alkylation of indoles and pyrrole with $\beta, \gamma$-unsaturated $\alpha$-ketoesters catalyzed by heteroarylidene-tethered bis(oxazoline) copper complexes. Chem. Commun. 2012, 48, 9281-9283. [CrossRef]

29. Palomo, C.; Oiarbide, M.; Kardak, B.G.; García, J.M.; Linden, A. Highly enantioselective friedel-crafts alkylations of pyrroles and indoles with $\alpha$ '-hydroxy enones under Cu(II)-simple bis(oxazoline) catalysis. J. Am. Chem. Soc. 2005, 127, 4154-4155. [CrossRef]

30. Bedekar, A.V.; Andersson, P.G. A new class of bis-oxazoline ligands for the cu-catalysed asymmetric cyclopropanation of olefins. Tetrahedron Lett. 1996, 37, 4073-4076. [CrossRef]

31. Liu, Y.; Zhou, X.; Shang, D.; Liu, X.; Feng, X. N, N'-dioxide-Scandium(III) complex catalyzed highly enantioselective Friedel-Crafts alkylation of indole to alkylidene malonates. Tetrahedron 2010, 66, 1447-1457. [CrossRef]

32. Chen, H.; Du, F.; Liu, L.; Li, J.; Zhao, Q.; Fu, B. Malonate-type bis(oxazoline) ligands with sp ${ }^{2}$ hybridized bridge carbon: Synthesis and application in Friedel-Crafts alkylation and allylic alkylation. Tetrahedron 2011, 67, 9602-9608. [CrossRef]

33. Zhou, J.; Ye, M.-C.; Huang, Z.-Z.; Tang, Y. Controllable enantioselective friedel-crafts reaction1 between indoles and alkylidene malonates catalyzed by pseudo- $\mathrm{C}_{3}$-symmetric trisoxazoline copper(II) complexes. J. Org. Chem. 2004, 69, 1309-1320. [CrossRef] [PubMed]

34. Son, S.; Fu, G.C. Nickel-catalyzed asymmetric negishi cross-couplings of secondary allylic chlorides with alkylzincs. J. Am. Chem. Soc. 2008, 130, 2756-2757. [CrossRef] [PubMed]

35. Evans, D.A.; Scheidt, K.A.; Fandrick, K.R.; Lam, H.W.; Wu, J. Enantioselective indole Friedel-Crafts alkylations catalyzed by bis (oxazolinyl) Pyridine-Scandium(III)triflate complexes. J. Am. Chem. Soc. 2003, 125, 10780-10781. [CrossRef] [PubMed]

36. Zheng, B.; Wang, M.; Li, Z.; Bian, Q.; Mao, J.; Li, S.; Liu, S.; Wang, M.; Zhong, J.; Guo, H. Asymmetric henry reaction catalyzed by a zn-amino alcohol system. Tetrahedron Asymmetry 2011, 22, 1156-1160. [CrossRef]

37. Zhu, S.-F.; Xu, B.; Wang, G.-P.; Zhou, Q.-L. Well-defined binuclear chiral spiro copper catalysts for enantioselective n-h insertion. J. Am. Chem. Soc. 2012, 134, 436-442. [CrossRef] [PubMed]

38. Lou, S.; Fu, G.C. Nickel/bis(oxazoline)-catalyzed asymmetric kumada reactions of alkyl electrophiles: Cross-couplings of racemic $\alpha$-bromoketones. J. Am. Chem. Soc. 2010, 132, 1264-1266. [CrossRef] [PubMed]

39. Kumari, P.; Bera, P.K.; Noor-ul, H.K.; Kureshy, R.I.; Abdi, S.H.; Bajaj, H.C. Asymmetric Friedel-Crafts addition of indoles to n-sulfonyl aldimines catalyzed by $\mathrm{Cu}(\mathrm{II})$ chiral amino alcohol based schiff base complexes. Catal. Sci. Technol. 2014, 4, 563-568. [CrossRef]

40. Ibáñez, I.; Kaneko, M.; Kamei, Y.; Tsutsumi, R.; Yamanaka, M.; Akiyama, T. Enantioselective Friedel-Crafts alkylation reaction of indoles with $\alpha$-trifluoromethylated $\beta$-nitrostyrenes catalyzed by chiral binol metal phosphate. ACS Catal. 2019, 9, 6903-6909. [CrossRef]

41. Islam, M.S.; Al Majid, A.M.; Al-Othman, Z.A.; Barakat, A. Highly enantioselective Friedel-Crafts alkylation of indole with electron deficient trans- $\beta$-nitroalkenes using Zn(II)-oxazoline-imidazoline catalysts. Tetrahedron Asymmetry 2014, 25, $245-251$. [CrossRef]

42. Singh, P.K.; Bisai, A.; Singh, V.K. Enantioselective Friedel-Crafts alkylation of indoles with nitroalkenes catalyzed by a bis(oxazoline)-cu (ii) complex. Tetrahedron Lett. 2007, 48, 1127-1129. [CrossRef]

43. Li, W. Chiral bis(oxazolinyl) thiophenes for enantioselective $\mathrm{Cu}(\mathrm{II})$-catalyzed Friedel-Crafts alkylation of indole derivatives with nitroalkenes. Catal. Lett. 2014, 144, 943-948. [CrossRef]

44. Tanaka, K.; Sakuragi, K.; Ozaki, H.; Takada, Y. Highly enantioselective Friedel-Crafts alkylation of n, n-dialkylanilines with trans- $\beta$-nitrostyrene catalyzed by a homochiral metal-organic framework. Chem. Commun. 2018, 54, 6328-6331. [CrossRef]

45. Li, Z.; He, M.; Xu, D.; Liu, Z. Graphene materials-based energy acceptor systems and sensors. J. Photochem. Photobiol. C. Photochem. Rev. 2014, 18, 1-17. [CrossRef]

46. Gao, J.-R.; Wu, H.; Xiang, B.; Yu, W.-B.; Han, L.; Jia, Y.-X. Highly enantioselective construction of trifluoromethylated all-carbon quaternary stereocenters via nickel-catalyzed Friedel-Crafts alkylation reaction. J. Am. Chem. Soc. 2013, 135, 2983-2986. [CrossRef]

47. Chen, J.-B.; Jia, Y.-X. Recent progress in transition-metal-catalyzed enantioselective indole functionalizations. Org. Biomol. Chem. 2017, 15, 3550-3567. [CrossRef]

48. Ono, N. The Nitro Group in Organic Synthesis; John Wiley \& Sons: Hoboken, NJ, USA, 2003; Volume 9.

49. Aitken, L.S.; Arezki, N.R.; Dell'Isola, A.; Cobb, A.J. Asymmetric organocatalysis and the nitro group functionality. Synthesis 2013, 45, 2627-2648.

50. Robinson, B. Alkaloids of the calabar bean. In The Alkaloids: Chemistry and Physiology; Elsevier: Amsterdam, The Netherlands, 1971; Volume 13, pp. 213-226.

51. Takano, S.; Ogasawara, K. Alkaloids of the calabar bean. In The Alkaloids: Chemistry and Pharmacology; Elsevier: Amsterdam, The Netherlands, 1990; Volume 36, pp. 225-251.

52. Greig, N.H.; Pei, X.F.; Soncrant, T.T.; Ingram, D.K.; Brossi, A. Phenserine and ring c hetero-analogues: Drug candidates for the treatment of alzheimer's disease. Med. Res. Rev. 1995, 15, 3-31. [CrossRef]

53. Berner, O.M.; Tedeschi, L.; Enders, D. Asymmetric michael additions to nitroalkenes. Eur. J. Org. Chem. 2002, $2002,1877-1894$. [CrossRef]

54. Calderari, G.; Seebach, D. Asymmetrische michael-additionen. Stereoselektive alkylierung chiraler, nicht racemischer enolate durch nitroolefine. Herstellung enantiomerenreiner $\gamma$-aminobuttersäure-und bernsteinsäure-derivate. Helv. Chim. Acta 1985, 68, 1592-1604. [CrossRef] 
55. Hayashi, T.; Senda, T.; Ogasawara, M. Rhodium-catalyzed asymmetric conjugate addition of organoboronic acids to nitroalkenes. J. Am. Chem. Soc. 2000, 122, 10716-10717. [CrossRef]

56. Choi, H.; Hua, Z.; Ojima, I. Highly enantioselective copper-catalyzed conjugate addition of diethylzinc to nitroalkenes. Org. Lett. 2004, 6, 2689-2691. [CrossRef]

57. Duursma, A.; Minnaard, A.J.; Feringa, B.L. Highly enantioselective conjugate addition of dialkylzinc reagents to acyclic nitroalkenes: A catalytic route to $\beta 2$-amino acids, aldehydes, and alcohols. J. Am. Chem. Soc. 2003, 125, 3700-3701. [CrossRef]

58. Czekelius, C.; Carreira, E.M. Catalytic enantioselective conjugate reduction of $\beta, \beta$-disubstituted nitroalkenes. Angew. Chem. Int. Ed. 2003, 115, 4941-4943. [CrossRef]

59. Watanabe, M.; Ikagawa, A.; Wang, H.; Murata, K.; Ikariya, T. Catalytic enantioselective michael addition of 1, 3-dicarbonyl compounds to nitroalkenes catalyzed by well-defined chiral ru amido complexes. J. Am. Chem. Soc. 2004, 126, 11148-11149. [CrossRef]

60. Li, H.; Wang, Y.; Tang, L.; Deng, L. Highly enantioselective conjugate addition of malonate and $\beta$-ketoester to nitroalkenes: Asymmetric $\mathrm{c}-\mathrm{c}$ bond formation with new bifunctional organic catalysts based on cinchona alkaloids. J. Am. Chem. Soc. 2004, 126, 9906-9907. [CrossRef]

61. Mellah, M.; Voituriez, A.; Schulz, E. Chiral sulfur ligands for asymmetric catalysis. Chem. Rev. 2007, 107, 5133-5209. [CrossRef]

62. Alammari, A.S.; Al-Majid, A.M.; Barakat, A.; Alshahrani, S.; Ali, M.; Islam, M.S. Asymmetric henry reaction of nitromethane with substituted aldehydes catalyzed by novel in situ generated chiral bis $(\beta \text {-amino alcohol-cu(oac })_{2} \cdot \mathrm{h}_{2} \mathrm{O}$ complex. Catalysts 2021, 11, 1208. [CrossRef]

63. Mao, J.; Nie, X.; Wang, M.; Wang, Q.; Zheng, B.; Bian, Q.; Zhong, J. Catalytic asymmetric nitroaldol (henry) reactions with copper(ii)/cyclopropane-based bisoxazoline complexes. Tetrahedron Asymmetry 2012, 23, 965-971. [CrossRef]

64. Blay, G.; Climent, E.; Fernandez, I.; Hernández-Olmos, V.; Pedro, J.R. Enantioselective henry reaction catalyzed with copper(II)-iminop yridine complexes. Tetrahedron Asymmetry 2007, 18, 1603-1612. [CrossRef]

65. Hao, X.-Q.; Xu, Y.-X.; Yang, M.-J.; Wang, L.; Niu, J.-L.; Gong, J.-F.; Song, M.-P. A cationic NCN pincer Platinum(II) aquo complex with a bis(imidazolinyl) phenyl ligand: Studies toward its synthesis and asymmetric Friedel-Crafts alkylation of indoles with nitroalkenes. Organometallics 2012, 31, 835-846. [CrossRef]

66. Wu, L.-Y.; Hao, X.-Q.; Xu, Y.-X.; Jia, M.-Q.; Wang, Y.-N.; Gong, J.-F.; Song, M.-P. Chiral NCN pincer Pt(II) and Pd(II) complexes with 1,3-bis(2'-imidazolinyl) benzene: Synthesis via direct metalation, characterization, and catalytic activity in the friedel-crafts alkylation reaction. Organometallics 2009, 28, 3369-3380. [CrossRef]

67. Schinnerl, M.; Seitz, M.; Kaiser, A.; Reiser, O. New applications of bis(oxazoline) ligands in catalysis: Asymmetric 1,2 and 1,4 addition of $\mathrm{znr}_{2}$ to carbonyl compounds. Org. Lett. 2001, 3, 4259-4262. [CrossRef] [PubMed]

68. Shintani, R.; Fu, G.C. Copper-catalyzed enantioselective conjugate addition of diethylzinc to acyclic enones in the presence of planar-chiral phosphaferrocene-oxazoline ligands. Org. Lett. 2002, 4, 3699-3702. [CrossRef] [PubMed]

69. Al Majid, A.M.; Islam, M.S.; Al-Othman, Z.A.; Al-Salhoob, A.F. Enantioselective additions of diethylzinc to aldehydes catalyzed by titanate(IV) complex with chiral bidentate bis-amide ligands based on cyclopropane backbone. Arab. J. Chem. 2017, 10, S964-S970. [CrossRef]

70. Mei, L.-y.; Yuan, Z.-1.; Shi, M. Chiral imidazoline-phosphine ligands for palladium-catalyzed asymmetric allylic substitutions. Organometallics 2011, 30, 6466-6475. [CrossRef]

71. Dugal-Tessier, J.; Dake, G.R.; Gates, D.P. Chiral phosphaalkene- oxazoline ligands for the palladium-catalyzed asymmetric allylic alkylation. Org. Lett. 2010, 12, 4667-4669. [CrossRef]

72. Sawada, T.; Nakada, M. Preparation of new chiral bisoxazoline ligands for the catalytic asymmetric intramolecular cyclopropanation of $\alpha$-diazo- $\beta$-keto phenyl sulfone to afford a useful bicyclo [3.1.0] hexane derivative. Tetrahedron Asymmetry 2012, 23, 350-356. [CrossRef]

73. Burguete, M.I.; Fraile, J.M.; García, J.I.; García-Verdugo, E.; Herrerías, C.I.; Luis, S.V.; Mayoral, J.A. Bis(oxazoline) copper complexes covalently bonded to insoluble support as catalysts in cyclopropanation reactions. J. Org. Chem. 2001, 66, 8893-8901. [CrossRef]

74. Jia, Y.-X.; Zhu, S.-F.; Yang, Y.; Zhou, Q.-L. Asymmetric friedel- crafts alkylations of indoles with nitroalkenes catalyzed by zn(ii)-bisoxazoline complexes. J. Org. Chem. 2006, 71, 75-80. [CrossRef]

75. Itoh, J.; Fuchibe, K.; Akiyama, T. Chiral phosphoric acid catalyzed enantioselective Friedel-Crafts alkylation of indoles with nitroalkenes: Cooperative effect of $3 \AA ̊$ molecular sieves. Angew. Chem. Int. Ed. 2008, 47, 4016-4018. [CrossRef]

76. Ganesh, M.; Seidel, D. Catalytic enantioselective additions of indoles to nitroalkenes. J. Am. Chem. Soc. 2008, 130, 16464-16465. [CrossRef]

77. Liu, H.; Du, D.M. Development of diphenylamine-linked bis(imidazoline) ligands and their application in asymmetric FriedelCrafts alkylation of indole derivatives with nitroalkenes. Adv. Synth. Catal. 2010, 352, 1113-1118. [CrossRef]

78. Buchcic, A.; Zawisza, A.; Leśniak, S.; Rachwalski, M. Asymmetric Friedel-Crafts alkylation of indoles catalyzed by chiral aziridine-phosphines. Catalysts 2020, 10, 971. [CrossRef]

79. Gao, M.-Z.; Wang, B.; Liu, H.-B.; Xu, Z.-L. Synthesis of chiral 2,5-bis(oxazolinyl)thiophenes and their application as chiral shift reagents for 1,1'-bi-2-naphthol. Chin. J. Chem. 2002, 20, 85-89. [CrossRef]

80. Yuan, Z.-L.; Lei, Z.-Y.; Shi, M. Binam and h8-binam-based chiral imines and Zn(OTf $)_{2}$ catalyzed enantioselective Friedel-Crafts alkylation of indoles with nitroalkenes. Tetrahedron Asymmetry 2008, 19, 1339-1346. [CrossRef] 
81. Meshram, H.M.; Kumar, D.A.; Reddy, B.C. Simple and efficient Friedel-Crafts alkylation of 1h-indole with electron-deficient alkenes promoted by zinc acetate. Helv. Chim. Acta 2009, 92, 1002-1006. [CrossRef]

82. Yongcheng, C.; Yuanyuan, C.; Zhengfeng, X.; Wenping, W. Friedel-Crafts reaction of indoles with nitroalkenes catalyzed by $\mathrm{Yb}(\mathrm{OTf})_{3}$. Chinese J. Org. Chem. 2011, 31, 1672-1677.

83. Liang, L.; Liu, Q.; Zhang, J.; Wang, F.; Yuan, Y. Efficient iron-catalyzed michael addition of indole to nitroolefins under solvent-free conditions. Res. Chem. Intermed. 2013, 39, 1957-1962. [CrossRef]

84. Jalal, S.; Sarkar, S.; Bera, K.; Maiti, S.; Jana, U. Synthesis of nitroalkenes involving a cooperative catalytic action of iron(III) and piperidine: A one-pot synthetic strategy to 3-alkylindoles, 2h-chromenes and n-arylpyrrole. Eur. J. Org. Chem. 2013, 2013, 4823-4828. [CrossRef] 